\title{
Corpus
}

$3 \mid 2004$

Usage des corpus en phonologie

\section{Présentation du volume. En quoi la phonologie est vraiment différente}

\section{Tobias Scheer}

\section{(2) OpenEdition \\ 1 Journals}

Édition électronique

URL : http://journals.openedition.org/corpus/193

DOI : $10.4000 /$ corpus. 193

ISSN : 1765-3126

Éditeur

Bases; corpus et langage - UMR 6039

Édition imprimée

Date de publication : 1 décembre 2004

ISSN : 1638-9808

Référence électronique

Tobias Scheer, «Présentation du volume. En quoi la phonologie est vraiment différente », Corpus [En ligne], 3 | 2004, mis en ligne le 02 décembre 2005, consulté le 08 septembre 2020. URL : http:// journals.openedition.org/corpus/193; DOI : https://doi.org/10.4000/corpus.193 


\title{
Présentation du volume En quoi la phonologie est vraiment différente
}

\author{
Tobias SCHEER \\ «Bases, Corpus et Langage », UMR 6039, Nice
}

\section{Introduction*}

Un collègue niçois, Marcel Vuillaume, m'a dit souvent que la phonologie, en comparaison avec la sémantique à laquelle il s'intéresse, a cet avantage d'être ancrée dans le réel : elle peut s'appuyer sur la phonétique et donc décrire les catégories qui sont pertinentes pour elle de manière objective. La pauvre sémantique, elle, doit se servir, pour la description de ses observables, de son propre objet d'étude. En conséquence, la circularité la guette en permanence. Les phonologues, conclutil, sont donc bien chanceux. Marcel a tort: la situation de la phonologie à l'interface avec la phonétique n'est pas un avantage mais au contraire un handicap grave. Elle a pour conséquence le fait que la phonologie, périodiquement dans son histoire et particulièrement aujourd'hui, est en proie à l'empirisme et a perdu, depuis une trentaine d'années maintenant, le contact avec le peloton de tête de la linguistique occupé par la syntaxe (alors que la phonologie a toujours été leader depuis le $19^{\text {ème }}$ siècle). La promesse d'objectivité dont la phonétique se sert pour enjôler les phonologues est l'obstacle majeur à l'étude des processus phonologiques cognitifs. Elle fait oublier que la Parole, objective, mesurable et à portée de main, n'est pas l'objet d'étude de la phonologie. Elle est un simple passage obligé sur le chemin qui mène à la découverte de la Langue, et n'a sur celle-ci aucun pouvoir ${ }^{1}$. Malgré cet

* Cet article doit beaucoup aux remarques, suggestions et censures de Sylvie Mellet, Pierre Encrevé et Sophie Wauquier.

1. «La Langue, distincte de la Parole, est un objet qu'on peut étudier séparément. [...] Non seulement la science de la Langue peut se passer

Corpus $n^{\circ} 3$ « Usage des corpus en phonologie » (2004), 5-84 
enseignement saussurien, la promesse « d'objectivité 》 a précipité, précipite et précipitera bien des phonologues dans les bras des sirènes phonétiques.

On peut être en accord ou en désaccord avec cette analyse. Elle aura au moins le mérite de montrer que parmi les disciplines linguistiques fondamentales, la phonologie est la seule qui doive se poser la question de son rapport avec la phonétique. Ni la syntaxe, ni la morphologie, ni la sémantique n'ont jamais été soupçonnées de subir une influence phonétique quelconque : il s'agit de disciplines qui décrivent des structures et processus exclusivement cognitifs, i.e. dont le théâtre est le cerveau et non pas la bouche ${ }^{2}$.

C'est donc ici une première spécificité de la phonologie: elle est seule à devoir définir le partage entre facteurs cognitifs et facteurs phonétiques avant même de pouvoir commencer le travail.

Dans l'introduction à ce volume, je voudrais établir la liste des spécificités phonologiques, et évaluer leur conséquence pour l'état d'avancement de la discipline. Anticipant le résultat, je dirai que le phonologue exerce un métier difficile, plus difficile en tout cas que les autres linguistes car il doit se battre perpétuellement pour définir son objet d'étude. Ou plutôt, si tous les linguistes doivent construire leur objet, le phonologue doit ajouter au travail de construction normal celui qui lui est imposé par les spécificités de sa discipline: une donnée phonologique est doublement ou triplement construite. Les embûches sont plus nombreuses et plus redoutables en phonologie qu'ailleurs. Dans ce cadre, je dégagerai quatre

des autres éléments du langage, mais elle n'est possible que si ces autres éléments n'y sont pas mêlés » (Saussure 1915 : 31).

2. La sémantique bien sûr est l'autre module qui assure l'interface avec la réalité extra-linguistique et extra-cognitive. Les tentations empiristes néanmoins semblent moindres ici, du fait justement que le sens est un objet assez insaisissable, en tout cas plus futile que la phonétique qui, elle, est bien concrète. Ceci n'empêche que la sémantique peut se trouver aux prises avec des forces empiristes : la pragmatique peut être conçue comme telle; elle est susceptible de faire oublier la Langue au profit de la Parole, attitude qui est cautionnée par la formule de Benveniste : «Nihil est in lingua quod non prius fuit in oratione ». 
propriétés phonologiques qui me semblent spécifiques à cette discipline. Il s'agit d'abord de tirer la ligne rouge de partage avec la phonétique ( $1^{\mathrm{er}}$ point), mais aussi avec le lexique, la diachronie et l'analogie ( $2^{\text {nd }}$ point, traité en sections 2 et 3 ). On note ensuite l'absence de récursivité, qui engendre le fait que le nombre des unités phonologiques est finie $\left(3^{\mathrm{e}}\right.$ point, traité en section 4). Enfin, je m'intéresserai aux conséquences, notamment pour les études à base de corpus, du fait que la phonologie semble être le seul domaine linguistique qui produise un système au sens structuraliste: on ne trouve l'équivalent du système phonématique dans aucune autre discipline ( $4^{\text {ème }}$ point, traité en section 5).

Cette introduction ouvre sur le problème phonétique puisque c'est tout de même celui qui en premier lieu constitue une pente naturelle, auréolée d'objectivité et d'assurance instrumentale. Il revient périodiquement et semble être indépendant des théories particulières: à chaque époque ses phonéticiens, qui pensent que la totalité de la variation sonore est due à un facteur physique et n'admettent aucune autre causalité. Les Kräuter (1877) et Meyer-Benfey (1901) sont aux néogrammairiens ce que la Phonologie Naturelle Générative ( $c f$. sections 2.3 à 2.6) est à SPE et le Inductive Grounding (Hayes 1999, Steriade 1997, Hayes et al. 2004) à la phonologie autosegmentale $^{3}$. C'est ainsi que le phonologue doit montrer perpétuellement que la phonologie existe, i.e. qu'elle n'est pas un simple appendice de la phonétique et qu'il est des processus cognitifs phonologiques qui ne doivent rien à ce qui se passe dans la bouche ${ }^{4}$. Cette phonologie, pour ne pas la confondre avec d'éventuels conditionnements multi-causals, est appelée

3. Il n'y que le structuralisme qui, je crois, a échappé au phonétisme, et pour cause: les phonéticiens structuralistes tels que Ilse Lehiste, Pierre Delattre ou encore Pierre Léon, en dehors de la démarche phonétique interne, ont toujours eu une attitude de validation et de contrôle lorsqu'il était question des relations avec la phonologie. Ils ont testé les hypothèses concernant le système phonologique (p. ex. Lehiste 1960) plutôt que de chercher à proposer des explications concurrentes. C'est ici une conséquence du caractère résolument abstrait (quoique non nécessairement cognitif) du structuralisme.

4. Ou aux gestes, lorsque la transmission est signée et non pas orale. 
autonome, ou «immaculée » selon le terme de Harris \& Lindsey (2000 : 187). Aucune autre discipline n'est dans cette position inconfortable.

Si les spécificités de la phonologie constituent en ellesmêmes un sujet intéressant, leur étude dans l'introduction à ce numéro de Corpus suppose qu'elles renseignent sur les particularités des corpus phonologiques. Je pense que c'est le cas, et m'emploierai à montrer ce lien dans les pages qui suivent. En particulier, je voudrais motiver la distinction entre corpus structuraliste et corpus générativiste.

Il est certainement vrai que cette introduction est (trop) longue ; ceci tient largement au sujet choisi. Le lecteur qui entend seulement profiter de la fonction classique d'une introduction - la présentation des contributions -, est invité à aller directement à la section 7, qui suit la conclusion (section 6).

\section{Construction de l'objet de la phonologie I : relations avec le lexique et la diachronie}

\subsection{Combien d'entrées lexicales ? Combien de règles?}

Soit l'alternance electri[k] - electri[s]ity en anglais (ou son équivalent français). Deux analyses sont envisageables, l'une «abstraite », l'autre " concrète ». La première pose, pour les deux formes de surface, une seule forme sous-jacente, à savoir celle qui présente le $[\mathrm{k}]$. A chaque fois que electri[s]ity est prononcé, le locuteur accède à l'entrée electri $[k]$ et $\mathrm{y}$ applique une règle qui transforme $[\mathrm{k}]$ en [s] devant [I], ou plus précisément devant [I] si celui-ci appartient au morphème -ity (ailleurs les vélaires restent intouchées, p.ex. king " roi », seek-ing « en demandant »).

La seconde analyse au contraire pose une entrée lexicale indépendante pour chacune des formes de surface. Lorsque electri[s]ity est prononcé, le locuteur fait exactement la même chose que lorsqu'il produit electri[k]: il accède directement au lexique. Aucune règle phonologique n'est appliquée afin d'obtenir ce résultat.

Les deux options ont des conséquences inverses sur le rapport entre la composante statique de la phonologie, i.e. le lexique, et sa composante computationnelle, i.e. les règles. La 
première minimise le lexique en maximisant le module procédural, alors que la seconde minimise la partie computationnelle et agrandit le lexique. Enfin, la première solution, au contraire de la seconde, installe un lien dérivationnel entre les deux formes.

\subsection{Le débat central des années 70 : l'abstraction}

Cet exemple illustre la question centrale de la phonologie post-SPE, l'abstraction, qui est posée dès 1968 par Kiparsky (1968a) : quelle distance entre une forme sous-jacente et sa réalisation de surface la théorie peut-elle admettre ? Dans le formalisme SPE, la mesure de cette distance est le nombre de règles qui s'appliquent au cours d'une dérivation.

Poser cette question était, dans l'après-SPE, un acte de civisme phonologique car l'application du modèle SPE allait bon train en faisant pousser des fleurs extravagantes. Il était un principe non explicite mais respecté par tous selon lequel la meilleure solution est celle qui suppose le plus petit lexique et la plus grande grammaire (i.e. le plus grand nombre de règles). Autrement dit, une seule forme sous-jacente était posée dès lors que deux ou plusieurs formes de surface entretenaient quelque rapport étymologique, paradigmatique ou sémantique. Etant donné deux formes entretenant un tel rapport, l'une était alors dérivée de l'autre, ou encore, si la forme sous-jacente ne coïncidait avec aucune forme de surface, les deux étaient dérivées d'un tiers objet (neutralisation absolue). Ce principe est parfois appelé d'économie cognitive, étiquette qui se discute car il promeut certes l'économie du volume lexical, mais augmente celui de la composante computationnelle. Or les deux, bien sûr, sont d'ordre cognitif.

Le générativisme se définit par le processus, contrairement au structuralisme qui met la structure, immobile par définition, en avant. Cette distinction est d'ailleurs fort utilement inscrite au fronton des deux maisons. Au-delà de l'exigence cognitive (le réalisme des objets théoriques) ou même de la Grammaire Universelle qui sont souvent citées comme les points de rupture entre structuralisme et générativisme mais ne peuvent guère exaucer cette ambition (Laks 1996, 1998, 2005, Encrevé 1997), l'opposition statique vs. 
procédural est sans doute ce qui véritablement distingue les deux approches (peut-être avec la transmission génétique de propriétés linguistiques). Il n'est pas surprenant, dans ces conditions, que l'introduction du procédural, dans un monde précédemment statique, se solde, dans un premier temps, par un excès de zèle : le générativisme flamboyant des années postSPE a réduit le lexique, partie statique s'il en est de la grammaire, à sa plus simple expression, et maximisé sa partie procédurale, i.e. la composante des règles ordonnées.

En l'espèce, on a vu ainsi certains phonologues soutenir sérieusement que soir et sérénade, oeil et oculaire, sweet et hedonistic, tooth et dental, thirst and torrid, thunder et detonation, queen et gynecology entretiennent un rapport dérivationnel et sont la manifestation de surface, en synchronie, d'une seule forme sous-jacente. Le spécialiste dans ce domaine était Theodore Lightner $(1978,1981)$, qui allait jusqu'à proposer sans aucune plaisanterie que la forme sous-jacente de tooth est en réalité $/ \mathrm{H}_{3} \mathrm{~d} /$ où $\mathrm{H}_{3}$ représente la laryngale indo-européenne qui colore en $o$. Partant de cette base lexicale, le locuteur anglais contemporain appliquerait, à chaque prononciation du mot tooth, la vocalisation des laryngales saussuriennes ainsi que, parmi une foule d'autres règles, la loi de Grimm et la loi de Verner.

Le problème ici n'est pas que quelque esprit trop zélé, ou trop conséquent, pousse le bouchon trop loin. Lightner n'était pas un cas isolé, loin de là ; mais bien des phonologues ne s'autorisaient pas pareille liberté. Ce qui donne à penser est que Lightner ait $p u$ le faire sans que la théorie ne lui oppose aucune borne, sans qu'il ne rencontre aucun obstacle, et que de surcroît, il soit invité à ainsi agir par le principe tacite invoqué supra, selon lequel ce monde sera meilleur si le nombre de règles augmente et que celui des entrées lexicales diminue.

D'où l'interrogation parfaitement légitime de Kiparsky (1968a) : n'y aurait-il pas moyen de trouver un principe formel qui tire une ligne rouge entre ce qui est recevable en matière d'abstraction, et ce qui dépasse les bornes? Le détail de ce débat ne peut être retracé ici, il est résumé ailleurs en plus grand détail (p.ex. Anderson 1985: 331 ssq, Kenstowicz \& Kisseberth 1977 : 1-62, 1979: 204ssq, Laks à paraître, $c f$. aussi Scheer 
Présentation. En quoi la phonologie est vraiment différente

2004 : §373). La recherche d'un tel principe formel salvateur a porté aussi bien sur le lexique que sur la partie procédurale. La Strict Alternation Condition agit sur l'abstraction du lexique en définissant les formes qui y sont admises en fonction des alternances de surface (elle met notamment au ban les neutralisations absolues, $c f$. Kiparsky 1968a,1973) $)^{5}$. La partie computationnelle de la grammaire a été gendarmée par l'importation en phonologie d'une notion syntaxique: Kean (1974), Mascaró (1976) et d'autres ont adapté la Strict Cycle Condition (Chomsky 1973). Enfin, les efforts de Kiparsky ont abouti, au début des années 80, à la mise en place de la Phonologie Lexicale (Kiparsky 1982a, b, 1985, Mohanan 1982, 1986, Rubach \& Booij 1984, Rubach 1984, 1993, Hargus \& Kaisse (eds.) 1993), modèle qui devait fournir l'architecture générale de la phonologie générative durant les années 80 . Outre le souci de limiter l'abstraction, la pierre angulaire de cette théorie est le fait que des règles de formation des mots, donc de la morphologie, peuvent être entrelacées avec des règles proprement phonologiques.

Mais, au bout du compte, il n'y avait toujours pas de mesure « objective » qui permette de déterminer si l'abstraction d'une analyse donnée est recevable ou non ; ni même, d'ailleurs, d'accord sur les critères qu'il convient d'appliquer pour déterminer le degré d'abstraction d'un scénario. En vrac, étaient proposées la simplicité (à géométrie variable bien sûr), la naturalité, l'élégance, la plausibilité phonétique, typologique (i.e. la marque) et psychologique (i.e. cognitive, "psychologique » est ici le mot employé classiquement depuis le $19^{\text {ème }}$ siècle). La chimère qu'on poursuivait s'appelait Evaluation Metric (ou Evaluation Measure), et la course, vaine et sans résultat, est retracée par exemple dans Kiparsky (1974), Hellberg (1978), Koutsoudas (1980), Dinnsen (1980), Campbell (1981), Goyvaerts (1981).

Les phonologues ainsi que les analyses continuaient donc à se partager en deux camps : les «abstraits» et les

5. Les effets de la Strict Alternation Condition sur le lexique sont exposés par exemple dans Rubach (1984: 7ss), Cole (1995: 73ss) et McMahon (2000: 35ss). 
« concrets $»^{6}$. Le second groupe érigeait en principe qu'entre deux analyses concurrentes, c'est toujours la plus concrète qui doit prévaloir. Le premier, au contraire, refusait catégoriquement ce type d'argument. Or cet affrontement était quelque peu factice, relevant surtout d'un état d'esprit, car le principe en or qui aurait permis de ranger chaque analyse dans un des deux tiroirs faisait défaut. Il fallait discuter de chaque cas individuellement, et le verdict était graduel plutôt que binaire : une analyse était plus ou moins abstraite, ou plus ou moins concrète. S'il y avait des cas parfaitement clairs qu'on pouvait classer à l'une ou l'autre des deux extrémités de l'échelle, comme par exemple les travaux de Lightner $(1978,1981)$ mentionnés plus haut (abstraits) ou ceux de la Phonologie Naturelle ( $c f$. ci-dessous, concrets), il subsistait une grande zone grise au milieu à propos de laquelle on pouvait épiloguer sans fin. Les cas classiques ici sont le velar softening et le trisyllabic laxening (ou trisyllabic shortening) en anglais. Le premier concerne l'alternance $[\mathrm{k}]$ - [s] dans electri $[k]$ - electri[s]-ity qui a servi d'illustration supra, et le second, la variation entre des voyelles longues et brèves dans des paires telles que div[aj]ne div[IInity, op[ej]que - op[æ] city.

\subsection{Recentrage diachronique du débat: la Phonologie Naturelle Générative}

Un autre angle d'attaque au problème de l'abstraction a été ouvert par la Phonologie Naturelle Générative ${ }^{7}$. La vraie

6. Schane (1968, 1974), Hyman (1970), Dell (1973), Gussmann (1980), Dresher (1981) par exemple représentent le premier, alors que Tranel (1981) et Leben \& Robinson (1977) plaident en faveur d'une phonologie concrète ( $c f$. aussi Kiparsky 1982c).

7. Il existe deux Phonologies Naturelles, l'une générative, l'autre nongénérative, qui sont toutes deux issues de la thèse de David Stampe (1972). La différentiation en deux branches s'est faite progressivement seulement, vers le milieu des années 70, et la ligne de fracture est grosso modo celle qui sépare l'approche formaliste (dont le modèle génératif est une variante) de l'approche fonctionnaliste du langage (dans le sens de Newmeyer 1998). La version non-générative de la Phonologie Naturelle est représentée, entre autres, par Dressler $(1974,1984)$, Hurch \& Rhodes (1996), Dziubalska-Kołaczyk (2001). Elle apprécie les événements phonologiques comme une manifestation de l'éternel combat entre le besoin de produire du contraste, nécessaire à la communication, et la 
Présentation. En quoi la phonologie est vraiment différente

question, dit-elle, est le rapport entre synchronie et diachronie. Certes, l'état synchronique d'une langue qui se présente à l'observateur renferme des processus, entre la structure sousjacente et la structure de surface, qui sont gérés in vivo par l'activité de la grammaire - donc du cerveau -, et qui se répètent à chaque prononciation des mots en question. Mais ce même état synchronique est aussi truffé de vestiges, de ruines, de traces, que d'anciens processus qui ne font plus partie de la grammaire ont laissés derrière eux (cf. à ce sujet la contribution de M.-J. Dalbera-Stefanaggi et J.-Ph. Dalbera). Poser la question de savoir si deux mots qui entretiennent un rapport étymologique, paradigmatique ou sémantique représentent deux entrées lexicales ou une seule revient donc en réalité à déterminer si le processus phonologique afférent est synchroniquement actif ou non.

La Phonologie Naturelle a ainsi exploré une perspective ouverte par Kiparsky (1968b), mais qui n'avait pas eu de réel écho dans les milieux proches de SPE, fixés comme on le sait sur la synchronie et ne faisant que peu d'état des données diachroniques. Radicalisant encore l'approche de Kiparsky (1968b), David Stampe dans sa thèse (Stampe 1972) et Theo Vennemann $(1972,1974 a, b)$ ont au contraire apprécié le problème de l'abstraction à travers le prisme de la diachronie : Vennemann (1976), faisant allusion aux excès à la Lightner, sermonne les phonologues au nom du «réalisme phonologique $»^{8}$. Nous allons voir dans la section suivante en quoi consiste exactement ce raisonnement diachronique et dans quelle mesure il déplace l'angle d'attaque du problème.

paresse (cérébrale, musculaire, etc.). Par ailleurs, elle nie l'existence d'un fonctionnement exclusivement interne, la Langue, qui ne doit rien à la Parole. La discussion menée ici concerne la seule Phonologie Naturelle Générative (dont les travaux seront introduits dans le texte principal).

8. Vennemann a toujours été un diachronicien invétéré. Il est une des rares figures de la grammaire générative qui ait produit une œuvre de première importance aussi bien dans le domaine classique de la philologie et de la diachronie (p.ex. Vennemann 1971, 1984, 1994, 1997) que dans celui de la théorie synchronique (p.ex. Vennemann 1972, 1978, 1988, 1991). 


\section{T. SCHEER}

\subsection{Le cycle de vie des règles : élimination, morphologisation et lexicalisation}

Dans le débat sur l'abstraction, la Phonologie Naturelle prend une position résolument anti-abstraite. Elle pense avoir trouvé la raison pour laquelle ce débat est seulement un sujet de discussion : SPE et ses adeptes ne font pas la différence entre la partie synchronique du fonctionnement linguistique et sa partie diachronique. Or, c'est ici qu'on trouvera le principe universel qui permet de déterminer si une alternance est abstraite ou concrète : c'est la ligne de partage des eaux tant recherchée sous le nom de Evaluation Measure (cf. supra). Les inadmissibles abstractions à la Lightner sont la conséquence du fait de vendre de la diachronie pour de la synchronie. Les ruines de vieilles règles, mortes depuis longtemps, ne font pas partie de l'objet d'étude de la phonologie: elles relèvent du lexique ou d'une autre composante, la morpho-phonologie ( $c f$. infra). Partant de ce constat, les alternances dites concrètes au contraire représentent exactement la partie synchronique du langage.

Cette position exprime la conception du changement qui est celle de la Phonologie Naturelle Générative. Les règles phonologiques ont une vie qui se déroule en cycles, ceux-ci étant toujours les mêmes, donc prédictibles. D'abord une règle naît - elle a alors une motivation phonétique et répond à la double pression que la loi du moindre effort et le désir de communiquer un contenu, et donc de produire du contraste, font peser sur le système. Toute altération d'un statu quo répond à une amélioration du rapport de force entre ces deux impératifs.

Dans sa jeunesse, la règle est vigoureuse et contrôle l'ensemble du lexique. Autrement dit, elle est sans exception ou, dans le vocabulaire qui sera introduit par la Phonologie Lexicale plus tard, post-lexicale. Toute séquence dans tous les mots de la langue qui correspond à la description structurale d'une règle est transformée selon les dispositions de son changement structural. A ce stade, la règle fait partie de la grammaire de la langue et codéfinit la compétence des locuteurs.

Ensuite, la règle vieillit. Elle se sclérose et perd progressivement le contrôle sur la totalité du lexique. Désormais, il peut y avoir des exceptions : toutes les séquences qui satisfont sa description structurale ne sont plus 
nécessairement transformées. Cette perte de vitesse se traduit typiquement par une lexicalisation des formes qui alternent, et par l'ajout de conditions morphologiques. Par exemple, toutes les vélaires d'une langue ne sont plus palatalisées devant voyelle antérieure; n'ont plus le pouvoir de transformer une vélaire, par exemple, que les voyelles antérieures qui se trouvent être le morphème du datif ou du diminutif. Ou encore, deux alternants qui ont précédemment été dérivés au moyen d'une règle à partir d'une forme sous-jacente unique représentent maintenant, après que la règle cesse d'exercer une coercition totale, deux entrées lexicales indépendantes. Par conséquent, celles-ci évolueront désormais indépendamment l'une de l'autre et non plus de manière concertée en fonction du devenir de la forme sousjacente commune. Ce sont les règles de l'évolution du lexique qui présideront à leur destin, règles qui souvent sont différentes de celles qui définissent des alternances globales.

En somme, donc, la Phonologie Naturelle Générative ne dit rien d'autre que ce que l'on enseigne dans tous les cours de phonologie diachronique : les règles (ou les lois) s'appliquent pendant une période précise - elles naissent à un moment donné et cessent d'être actives à un moment donné. Autre exemple, la vocalisation de 1 en Coda interne en français est un processus mort depuis fort longtemps : les mots qui étaient présents dans la langue du temps de son activité (i.e. en ancien français au moins) ont tous perdu leurs 1 en Coda au profit d'un [w]. D'où les alternances cheval - chevaux etc. Certains ont pu restaurer, après la mort de la règle, le 1 ancien ( $c f$. malgré < afr maugré), ou ont un comportement flottant aujourd'hui : c'est le cas de caporal par exemple $\left(<16^{\text {ème }}\right.$ italien caporale $)$ qui, selon les locuteurs, fait caporaux ou caporals. Il est exclu, en revanche, qu'un mot qui n'ait pas été présent du vivant de la règle, comme chacal, rital ou carnaval par exemple, soit transformé par elle : les pluriels sont seulement chacals, ritals et carnavals.

Il peut arriver, cependant, qu'un mot soit transformé quand bien même il est entré dans la langue après la bataille. Dans le cas de notre exemple, cela produit un pluriel comme 
terminaux, du singulier terminal ${ }^{9}$. Ce qui est capital alors, c'est que la force qui porte le singulier terminal à terminaux n'est pas phonologique, ni ne relève de la grammaire ${ }^{10}$. Il s'agit d'un processus bien réel mais qui ne fait pas partie de la compétence (ou de la Langue). Au $19^{\text {ème }}$ siècle, on appelait ce processus, l'analogie, "psychologique ».

\subsection{La compétence est seulement synchronique - la diachronie et ses ruines morphologisées ne sont pas notre affaire}

Résumons-nous. Selon la Phonologie Naturelle Générative, toutes les règles jeunes sont motivées uniquement par un conditionnement phonétique. Celui-ci répond à un avantage fonctionnel qui se calcule en croisant la loi du moindre effort et le désir de produire du contraste. Ces règles font partie de la compétence du locuteur au sens chomskyen (de la Langue en vocabulaire saussurien) et s'appliquent sans aucune exception. En vieillissant, elles acceptent des conditions d'application supplémentaires qui peuvent être de nature morphologique ou lexicale. Les règles cessent alors d'être actives phonologiquement: dans l'architecture générale de la grammaire que propose la Phonologie Naturelle Générative, elles passent du module phonologique dans le module morphophonologique. Ce transfert les élimine de la compétence proprement phonologique. Elles peuvent se scléroser davantage encore lors de leur vie dans le module morpho-phonologique de

9. On peut être témoin de la forme terminaux à l'aéroport de Nice qui est structuré en deux termin... Chacun voit bien qu'il existe une réticence à prononcer ce mot au pluriel; en passant outre, le résultat peut partir dans les deux sens. Un poids analogique très important pèse aussi sur les adjectifs en -al empruntés après la mort de la règle tels que banal, nasal, naval. La norme, souvent avec peu de fortune, essaie de maintenir les pluriels banals, nasals, navals.

10. Selon le sens qu'il revêt dans les différentes théories, le mot «grammaire» peut être sujet à caution ici : certains comme Antoine Culioli par exemple peuvent opérer un découpage où l'analogie, activité « épilinguistique », cohabite avec les règles (les « lois phonétiques » des néogrammairiens) au sein de la grammaire (qui serait subjective dans le cas de l'analogie). Quel que soit le découpage et les termes en usage, tous s'accordent qu'il existe une différence de principe entre les deux types de forces qui pèsent sur la chaîne sonore. 
telle sorte qu'elles meurent complètement et ne laissent derrière elles que des vestiges lexicaux. Ce sont des ruines de ce type que nous observons probablement en français moderne : cheval et chevaux sont deux entrées lexicales différentes, aucune dérivation n'a lieu, ni aucune règle n'est appliquée lorsqu'un locuteur prononce chevaux.

Dans ces conditions, on est très loin du mot d'ordre tacite donné par SPE selon lequel toutes les formes qui entretiennent un rapport étymologique, paradigmatique ou sémantique ont pour base une seule forme sous-jacente. Et que la meilleure grammaire est celle qui a le plus petit lexique et la plus grande composante procédurale. Les analyses « synchroniques » de Lightner ( cil - oculaire, thirst - thunder etc.) par exemple, aux yeux de la Phonologie Naturelle Générative, ne sont qu'une mauvaise plaisanterie. Tous les «alternants» de ce type représentent des entrées lexicales indépendantes et ne sollicitent aucune activité grammaticale lors de leur prononciation.

Aussi, la période post-SPE produisait des analyses « synchroniques » qui étaient censées représenter la compétence des locuteurs mais accouchaient systématiquement d'une séquence de règles ordonnées qui reproduisait fidèlement les événements diachroniques des cinq ou dix siècles précédents. Pour la Phonologie Naturelle Générative, cette étrange coïncidence n'est qu'une conséquence supplémentaire du refus de faire le tri entre les processus synchroniques et diachroniques. La grammaire que le linguiste est appelé à écrire doit rendre compte de la seule compétence (de la seule Langue) des locuteurs. C'est ici la mission assignée aussi bien par Saussure que par Chomsky (la dernière phrase du Cours affirme que « la linguistique a pour unique et véritable objet la langue envisagée en elle-même et pour elle-même »). Or à l'évidence les sujets qui vivent au $20^{\text {ème }}$ siècle n'ont pas connaissance des règles qui s'appliquaient du temps des Carolingiens.

Dans cette perspective, les événements diachroniques ne regardent pas le phonologue, et les régularités morphophonologiques font partie d'un module de la grammaire qui est différent de la phonologie. On reconnaît ici sans aucune peine la filiation, non assumée d'ailleurs, avec le structuralisme 
américain le plus classique où la séparation des niveaux de description (phonétique, phonémique, morphémique) est centrale ( $c f$. Laks à paraître sur cette filiation). C'est encore ici un croche-pied fait à SPE qui, justement, avait aboli toute distinction de niveaux, à part celle qui existe entre la structure sous-jacente et la structure de surface.

Une conséquence immédiate de cette attitude est un phonétisme parfait: la seule cause pour les alternances phonologiques est un conditionnement phonétique car seules les règles synchroniquement actives définissent la compétence (la Langue). Or celles-ci ont comme seule motivation la phonétique. Le nom de la théorie qui met en avant l'adjectif «naturel» vient d'ailleurs de là : est naturel ce qui est phonétique, et seulement ceci. Est non-naturel ce qui n'a pas de motivation phonétique. Le phonologue ne doit décrire que ce qui est naturel.

\subsection{Le principe en or qui tranche le débat sur l'abstraction : la transparence phonétique}

$\mathrm{Au}$ nom du réalisme phonologique (notons la différence par rapport au réalisme cognitif) et du précepte chomskyen et saussurien selon lequel sinon le seul, du moins le premier objet d'intérêt en linguistique est la compétence (la Langue), la Phonologie Naturelle Générative élimine la majeure partie des alternances qui font l'objet de l'étude phonologique classique (et de type SPE). C'est la position radicalement inverse du toutprocédural SPE: l'existence d'un rapport étymologique, paradigmatique ou sémantique entre deux formes est une condition nécessaire, mais nullement suffisante pour les faire dériver d'un objet sous-jacent commun. La condition suffisante est la naturalité, et celle-ci se définit phonétiquement: est naturel ce qui 1) a une motivation phonétique, 2) n'a pas d'exceptions et est donc surface-true et 3) n'est sujet à aucun conditionnement morphologique ou syntaxique. Le produit de ces conditions est l'ensemble des processus qui ont lieu en ligne dans le cerveau du locuteur à chaque fois que celui-ci prononce un mot : les processus synchroniquement actifs. Il coïncide avec la compétence phonologique. 
Ceci n'est pas à dire que la Phonologie Naturelle nie l'existence d'alternances qui, tout en présentant un conditionnement morphologique, sont parfaitement régulières et productives, donc relèvent de la compétence. Un exemple en est le velar softening (electri $[k]$ - electri[s]ity) qui a été mentionné plus haut. Selon la Phonologie Naturelle Générative, ce type de phénomène est géré par un autre module de la grammaire, le module morphophonologique. Les règles phonologiques $(P$ rules) font référence aux seules propriétés phonétiques, alors que les règles morphophonologiques (MP rules) comportent nécessairement, dans leur description structurale, une condition morphologique.

Enfin, il y a une catégorie supplémentaire de règles, les via-rules, qui gèrent la supplétion : keep [kiip] et kept [kept] par exemple sont certes tous deux rejetés dans le lexique car l'alternance [ii] - $[\mathrm{E}]$ n'a rien de naturel et présente comme condition le rapport présent - prétérit (et encore ne survient pas dans tous les verbes dont le présent est en [ii] : seek - seeked etc.), mais pour autant ces deux formes entretiennent une relation grammaticale. Car la paire keep - kept n'a certainement pas le même statut lexical que le couple table - maison. Il y a donc un diacritique qui fait partie de l'entrée lexicale de keep et pointe vers l'entrée lexicale kept. Ce diacritique et le processus qui relie les deux formes est appelé via-rule (Hooper $1976: 47 \mathrm{sq})^{11}$.

Dans le paysage de la Phonologie Naturelle Générative, la composante phonologique traite donc des seules alternances qui sont phonétiquement motivées. Il s'agit là d'une charge de travail qui représente une petite minorité seulement de ce que SPE demande à la composante phonologique de gérer : on peut penser que $80 \%$ à $90 \%$ des alternances dont s'occupe SPE se trouvent ainsi éliminées de la phonologie, reléguées dans le module morpho-phonologique ou dans le lexique.

La Phonologie Naturelle est donc une phonologie minimaliste. Elle pense avoir trouvé le principe en or dont il a été question plus haut et que les autres phonologues de la période post-SPE ont recherché en vain : pour savoir si une

11. Ce concept est également développé (sans référence aux via-rules d'ailleurs) dans le modèle de la représentation lexicale de Kaye (1989, 1995) (cf. Scheer ms). 
alternance est suffisamment peu abstraite pour faire dériver ses membres d'une forme sous-jacente unique, il suffit de savoir si elle est synchroniquement active. Or, une alternance est gérée en ligne par la partie computationnelle si et seulement si elle remplit les trois conditions sus-mentionnées : 1) elle ne doit pas avoir d'exceptions, 2) elle ne doit être conditionnée par aucun facteur morphologique ou syntaxique, 3) elle doit avoir comme seule motivation la phonétique.

Cet ensemble de conditions a été déposé dans deux principes qui, il est vrai, ne s'ensuivent pas nécessairement de ce qui vient d'être dit. Ils n'en sont pas moins la mesure " officielle », en Phonologie Naturelle Générative, de ce qui a le droit de cité dans le module phonologique. Il s'agit de la True Generalization Condition (Hooper 1976: 13ssq) et de la NoOrdering Condition (Hooper 1976: 18ssq). La première demande à ce que toute règle phonologique soit phonétiquement transparente: une règle peut faire appel aux seules informations qui sont présentes dans le signal phonétique. Ceci exclut, bien sûr, tous les cas d'opacité, c'est-à-dire de dérivations où l'effet d'une règle donnée est altéré par une règle subséquente et ainsi rendu méconnaissable en surface. Mais la No-Ordering Condition va plus loin : non seulement le module phonologique est incapable de gérer l'opacité, mais encore tous les cas où une règle est nécessairement ordonnée avant ou après une autre règle sont mis au ban de la phonologie quand bien même le résultat est transparent en surface.

En somme, donc, il existe un niveau sous-jacent qui est relié à un niveau de surface au moyen de règles. Une dérivation peut nécessiter plus d'une règle afin de produire le résultat phonétique, mais à condition que celles-ci s'appliquent simultanément (ou dans n'importe quel ordre).

La Phonologie Naturelle apporte donc une solution radicale à la question de l'abstraction et, partant, à celle de la surgénération et de l'arbitraire du conditionnement contextuel, failles majeures de SPE. Car la distance maximale entre forme sous-jacente et forme de surface, calculée en pas dérivationnels, est désormais de un : les formes intermédiaires sont éliminées. C'est en effet ce qu'on peut appeler réduire l'abstraction. La surgénération et l'arbitraire sont également traités puisque la 
Présentation. En quoi la phonologie est vraiment différente

seule cause, en phonologie, est désormais phonétique (sic). Une vélaire ne peut palataliser devant voyelle postérieure ou [p] parce que ces articulations n'attirent pas vers le locus palatal.

\subsection{Pourquoi la Phonologie Naturelle Générative a décliné}

On le voit, tous les chemins mènent à la phonétique : la composante phonologique est faite de phonétique, et de phonétique seulement. C'est ainsi que ce paradoxe se trouve au terme d'un questionnement parfaitement légitime, nécessaire et salutaire. Ceux qui entendent réparer un excès, celui de l'abstraction, tombent, chemin faisant et sans trop s'en rendre compte, mais inéluctablement, dans l'excès inverse, le phonétisme. Certes ce mouvement de balancier n'est guère extraordinaire dans l'histoire de la linguistique, $c f$. Anderson 1985 (et dans l'histoire tout court d'ailleurs), mais il ne témoigne pas d'un sens aigu pour la cumulativité (Encrevé 1997, Durand \& Laks 1996). Si la phonologie se résume à de la phonétique ${ }^{12}$, on est en droit de se demander si le remède n'est pas pire que le mal.

C'est pour cette raison en partie, je pense, que la Phonologie Naturelle Générative a disparu du paysage phonologique assez rapidement et se trouve, aujourd'hui, sans descendance moderne. ${ }^{13}$ Après une période initiale, l'expression la plus compacte de la Phonologie Naturelle, encore indifférenciée entre les versions générative et non-générative, se trouve dans les actes de la Parasession on Natural Phonology (Bruck et al. 1974). Hooper (1973, 1974, 1975) propose ensuite un résumé informé de la nouvelle théorie et signe le livre qui en deviendra la référence (Hooper 1976). Enfin, la thèse de

12. Sujet d'actualité, car c'est en somme ce que propose la branche de la théorie de l'optimalité qui promeut le Inductive Grounding (Hayes 1999, Steriade 1997, Hayes et al. 2004). A cette différence près, toutefois, que la Langue continue à exister formellement : le contenu des contraintes est exclusivement extra-phonologique (notamment phonétique, mais aussi fonctionnel ou psycho-linguistique), mais leur ordonnancement (dont la seule fonction est de décrire la variation paramétrique rencontrée dans les langues) est décidé par la phonologie autonome (cf. Scheer 2004 : §319).

13. Nonobstant le fait que des courants modernes tel le Inductive Grounding (Hayes 1999, cf. note 12) peuvent se réclamer de la Phonologie Naturelle Générative : ils n'en sont pas issus mais la redécouvrent. 


\section{T. SCHEER}

Patricia Donegan (1978) ainsi que les articles de Donegan \& Stampe $(1978,1979)$ représentent la fin de la période que la Phonologie Naturelle Générative a marquée de son sceau.

Laks (à paraître) fait l'histoire de cette théorie en plus grand détail et montre sa fonction dans le devenir de la phonologie générative. Il fait remarquer, notamment, que la fin de la période de gloire de la Phonologie Naturelle Générative coïncide avec l'installation, dans la lignée plus étroitement chomskyenne, du nouvel outil qui en devait changer profondément la face et incarnait l'espoir d'un antidote formel à l'abstraction: l'autosegmentalisme (Goldsmith 1976, Kahn 1976). De même, je l'ai dit supra, les efforts de Paul Kiparsky ont abouti, au tout début des années 80 avec la naissance de la Phonologie Lexicale, au modèle qui donna l'architecture générale dans laquelle les diverses structures autosegmentales se sont insérées.

Tout cela est certainement vrai : le remusclage des concepts purement formels au sein de la lignée chomskyenne SPE, ainsi que les espoirs associés de vaincre la surgénération ${ }^{14}$, étaient autant de causes externes qui ont fait que la Phonologie Naturelle Générative a perdu de son mordant, et donc de la vitesse. L'arrivée des nouveaux outils formels a en quelque sorte détruit son fonds de commerce.

Mais je pense que là n'est pas la seule raison de son déclin : il y a aussi une cause interne. Cette cause interne est précisément le phonétisme, point final auquel, je pense, la contestation initiale, parfaitement fondée et nécessaire, a dû mener inéluctablement dès lors que ce n'était pas les effets néfastes de l'abstraction qui étaient en ligne de mire, mais l'abstraction elle-même.

En somme, donc, l'histoire de la Phonologie Naturelle Générative est un cas d'école illustrant comment une théorie linguistique glisse petit à petit dans des sphères extrasaussuriennes où la Langue d'abord n'existe plus, ensuite est

14. Qui étaient, et sont, parfaitement fondés : l'interdiction de croiser les lignes d'association était un succès en la matière. Or cette restriction n'a pu être conçue que dans un cadre autosegmental (cf. Scheer $2004: \S 308$ sur le caractère non-intentionnel de cet effet de l'autosegmentalisme). 
explicitement niée. La théorie se précipite ainsi dans les bras du phonétisme et de ce fait perd sa légitimité ${ }^{15}$. C'est l'histoire du bébé et de l'eau du bain, que Paul Kiparsky par exemple a toujours clairement appréciée en tant que telle : il a essayé de jeter l'eau abstraite tout en préservant le bébé, lui aussi abstrait.

\subsection{La syntaxe et la sémantique ne doivent se battre ni avec le lexique ni avec la diachronie}

Il est évident que les phonologues (et, il est vrai, les morphologues) sont seuls à devoir mener le débat concernant l'objet de leur étude avant de pouvoir procéder à quelque analyse que ce soit : phonétique, lexique, diachronie.

La syntaxe et la sémantique ne sont pas contraintes à construire leur objet de cette façon: il est garanti que toute phrase prononcée par un sujet est le résultat d'une activité cognitive procédurale en ligne. Car le lexique ne contient pas des phrases entières: chaque phrase a nécessairement été construite par la grammaire in vivo, c'est-à-dire de manière synchronique $^{16}$. Ceci vaut pour sa structure autant que pour ses

15. Une autre théorie phonologique qui a suivi la même pente, et avec un résultat social similaire, est la Phonologie Déclarative (Scobbie et al. 1996, Coleman 1995, 1998). Un de ses fondateurs, John Coleman, nie désormais l'existence de la phonologie tout court, qu'il relègue au statut d'affabulations non-scientifiques: "Are there any phonological phenomena which cannot be described or formulated using phonetic representations, and which require us also to employ more abstract, phonological representations? [...] Theory-internal philosophical argumentation alone is not sufficient. [...] In short, the study of grammar must rest on good science, not on philosophy » (italiques d'origine) (Coleman 2002 : 97). Il est vrai, cependant, que tous ceux qui se réclament de cette théorie n'ont pas suivi Coleman sur ce point (Angoujard 1997). La grande masse de la théorie de l'optimalité, suivant le Inductive Grounding ( $c f$. note 12), s'est également engagée sur la pente du phonétisme, avec un bilan scientifique et social encore incertain, mais au sujet duquel le présent texte fait une prédiction.

16. Le courant néo-behaviouriste dit «usage-based» ou «exemplariste», dont le cheval blanc est Joan Bybee (2001) (il s'agit en fait de Joan Hooper que nous avons rencontrée en Phonologie Naturelle Générative, qui désormais a repris son nom de jeune fille), nie cette évidence. Il n'y a aucune raison, je pense, de s'attarder à ce retour à une linguistique présaussurienne, mécaniste et à la vapeur, qui du reste professe sans aucune plaisanterie que les procédés cognitifs qui permettent à un humain 
effets sémantiques. Il est vrai qu'il existe des cas où une séquence de mots doit ses propriétés syntaxiques et sémantiques non pas à l'activité grammaticale en ligne mais au lexique : ce sont les expressions figées du type « casser sa pipe ». Or cellesci, en comparaison avec l'étendue de l'équivalent phonologique, d'une part constituent la portion congrue et d'autre part se trahissent clairement par leur comportement. Tout le monde s'accorde qu'il faut reléguer ces expressions dans le lexique, qu'elles sont donc enregistrées telles quelles, et leur reconnaissance n'a jamais nécessité de débat sur l'architecture générale de la grammaire (composante phonologique, morphophonologique) ni sur leur proportion dans la masse totale des faits dont le linguiste doit rendre compte.

La même chose est vraie pour la diachronie : en dehors de ces expressions figées, il n'y a pas de ruines léguées par des processus ou des états d'autrefois. Il est garanti que la syntaxe et la sémantique de chaque phrase ont été fabriquées au moment même de sa prononciation.

Le cas de la morphologie est intéressant car il relève, je pense, des deux domaines : la flexion se comporte comme la syntaxe et la sémantique, alors que la dérivation rencontre les mêmes écueils que la phonologie. Aucun linguiste n'est probablement prêt à accepter l'idée que des morphèmes flexionnels sont enregistrés avec des radicaux ou d'autres morphèmes dans la même entrée lexicale. En revanche, la question de savoir si band-age est le résultat d'une concaténation morphologique ou au contraire constitue une seule entrée lexicale se pose.

La phonologie est donc seule, avec la morphologie dérivationnelle, à devoir débattre de son objet d'étude avant de pouvoir commencer l'analyse. Il est probable qu'une grande partie des alternances qu'un observateur naïf, ou SPE en l'occurrence, identifierait, en réalité ne relève pas d'une activité phonologique computationnelle in vivo, mais du lexique et de la supplétion. La ligne rouge se trouve sans doute quelque part entre les deux extrêmes, constitués respectivement par le

d'apprendre à jouer au piano sont nécessaires et suffisants pour l'acquisition de sa langue maternelle (Bybee 2001 : 14). 
Présentation. En quoi la phonologie est vraiment différente

fantaisiste Lightner et la phonétiste Phonologie Naturelle Générative. Aussi, toute l'encre versée ne nous a guère avancés quant à sa localisation précise et formelle : il n'y a toujours pas d'aune qui mesure le degré d'abstraction (evaluation measure), et encore moins de limite ferme dessinée dans le continuum entre « abstrait» et « concret ».

Mais le débat sur l'abstraction aura servi au moins à une chose : nul ne peut plus prétendre qu'il ne sait pas. Que, par ignorance, négligence ou refus d'admettre qu'il existe des « alternances » lexicalisées, il saute l'étape de la construction de son objet d'étude. Tout phonologue sait désormais qu'il exerce un métier difficile qui suppose une activité préparatoire préalable à l'analyse elle-même, et qu'à défaut de ce raisonnement, ses résultats risquent d'être nuls et non-avenus.

Ceci étant dit, hélas, l'amnésie semble frapper les phonologues qui, dès les années 80 et l'avènement de la Phonologie Lexicale, mais surtout depuis qu'ils pratiquent la théorie de l'optimalité ${ }^{17}$, ont tendance à ne plus se souvenir du débat sur l'abstraction et de celui autour de la Phonologie Naturelle Générative. Ainsi Borowsky (2000 : 9) dérive le plus naturellement du monde et sans aucune discussion ni aucun avertissement fifth, width, thieves, wolves, description, scripture, conception, reception de five, wide, thief, wolf, describe, scribe, conceive, receive. C'est ici seulement un point de continuité entre SPE et la théorie de l'optimalité (Hulst \& Ritter 2000 en proposent une liste plus conséquente), qui en l'occurrence fait comme si les années 70 et 80 n'avaient pas existé. L'absence de cumulativité dans le sens d'Encrevé (1997) et Durand \& Laks (1996), encore ici, n'est pas bon signe pour la discipline : celle-ci, semble-t-il, n'a toujours pas pris acte de son statut spécifique.

17. Les analyses faites au sein de cette théorie sont particulièrement empreintes d'amnésie, mais bien sûr celle-ci est aussi visible dans d'autres approches telles la Phonologie Déclarative ou la Phonologie de Gouvernement. 


\section{Construction de l'objet de la phonologie II : la syntaxe et la sémantique ne doivent pas se battre avec l'analogie non plus}

L'analogie est un autre facteur coutumier en phonologie et en morphologie dérivationnelle (découpage désormais familier) mais inconnu ailleurs. Elle a été illustrée plus haut par le pluriel terminaux (du singulier terminal). Il y aurait beaucoup à dire à ce sujet: son histoire, ses différentes formes (intraparadigmatique, inter-paradigmatique), son abus ${ }^{18}$, ses incarnations dans les différentes théories, néogrammairienne jusqu'aux Output-Output Constraints de la théorie de l'optimalité. Chaque linguiste, phonologue ou non, connaît le dossier car il a rencontré l'analogie... où ? en phonologie, précisément. Ce constat suffirait seul à résumer mon propos ici : seule la phonologie et la morphologie dérivationnelle doivent prendre en compte ce facteur.

Dans la conception classique néogrammairienne (p.ex. Paul 1880), il y a deux forces en phonologie, de nature différente et ayant l'effet opposé : les lois phonétiques détruisent l'uniformité du paradigme et plus généralement la régularité dans la langue, alors que l'analogie œuvre pour leur rétablissement ${ }^{19}$. Les lois phonétiques sont aveugles et ne s'inquiètent pas de ce que la moitié seulement d'un paradigme ou d'une classe de verbes soit transformée. Elles constituent le véritable objet d'étude en phonologie, la Langue en termes saussuriens, la compétence chez Chomsky. Le combat fondateur des néogrammairiens a été, justement, de montrer qu'elles fonctionnent exactement comme d'autres lois naturelles dans d'autres disciplines telle la biologie, la chimie ou la physique, et que la linguistique est donc une discipline scientifique et non philologique. Par conséquent, les lois phonétiques s'appliquent à toute cible potentielle et ne souffrent aucune exception : leur effet

18. Ehonté et fréquent en diachronie. Pour aller vite, lorsque le diachronicien ne sait pas expliquer telle ou telle forme, même parfois $80 \%$ des formes, il les met sur le compte d'une activité analogique qui échappe, par définition, aux critères habituels fondés sur la prédiction et la réfutation. L'analogie a le dos large et ne coûte pas cher.

19. La contribution de M.-J. Dalbera-Stefanaggi et J.-Ph. Dalbera illustre ce propos à la lumière de la conception structuraliste. 
est prédictible et, partant, les propositions, falsifiables. On le sait, ceci est une propriété indispensable pour toute théorie qui veut prétendre au statut scientifique.

L'ennemi des lois phonétiques est l'analogie: elle est imprédictible, a l'effet inverse et surtout procède d'un mécanisme différent. L'analogie ne transforme pas une séquence si et seulement si elle y rencontre un certain contexte. Elle modifie un objet non pas en fonction d'un plan pré-établi (la condition contextuelle), mais au hasard de ce que le lexique de la langue en question offre : la transformation est le résultat d'une règle de quatrième proportionnelle où un objet seul ou plusieurs formes entretenant un lien grammatical sont rendus similaires à une unité lexicale différente ou d'autres formes entretenant le même rapport grammatical. La mémoire y joue un rôle évident : dans le cas du pluriel terminaux, c'est parce que le pluriel n'a jamais été utilisé et est donc absent du lexique (les paires cheval - chevaux etc. sont des entrées lexicales indépendantes, $c f$. supra) que le locuteur doit en fabriquer un. Il pourrait pour ce faire simplement ne pas varier (terminal terminals), mais en l'occurrence c'est la présence dans la langue d'un certain nombre de paires comme cheval - chevaux dont le singulier se termine en -al qui impose sa loi : le locuteur va reproduire leur fonctionnement.

Chaque production de pluriel pour des mots sans pluriel lexicalisé est particulière et peut aboutir à une forme régulière en -als ou une forme analogique en -aux. Ce choix peut varier de locuteur à locuteur, et même d'occurrence à occurrence du même mot chez le même locuteur. Par conséquent, rien ne peut être prédit et, partant, aucune analyse analogique ne peut jamais être réfutée.

C'est la raison pour laquelle les néogrammairiens appelaient les forces sûres et prédictibles qui agissent sur la langue des lois, par opposition au phénomène "psychologique» de l'analogie. Le mot psychologique a été choisi visiblement afin de montrer le rôle de facteurs extragrammaticaux comme la mémoire, la fréquence et une certaine liberté de décision (qui certes n'est pas consciente). L'article de Joaquim Brandão de Carvalho dans ce volume étudie un de ces 
aspects de l'analogie: il enquête sur l'influence qu'a la fréquence lexicale sur l'analogie.

L'analogie accompagne donc le phonologue dès ses premiers pas, et ne le quitte plus. Elle lui tend bien des pièges, car si certaines formes avouent leur raison d'être analogique d'emblée, il est parfois difficile, sinon impossible, de déterminer si une forme est née sous l'empire d'une force grammaticale ou analogique.

Comme le lexique et la diachronie, le partage du gâteau phonologique en territoire grammatical et analogique est une question perpétuelle à laquelle le phonologue doit répondre avant de pouvoir entamer l'analyse. S'il se méprend en proposant une solution qui mobilise des concepts grammaticaux pour l'explication d'un objet analogique, il aura perdu son temps.

Or en syntaxe et en sémantique (ainsi qu'en morphologie flexionnelle je pense), nul n'est encore tombé dans le piège analogique. L'analogie n'y a pas cours. Elle ne peut y faire son marché car elle s'appuie sur des unités lexicales afin de construire le modèle auquel elle va conformer l'unité à construire $^{20}$. Par ailleurs, le rôle de la mémoire dans les processus analogiques a été mentionné : ce n'est qu'en cas de défaillance à la fois des procédés grammaticaux et de l'enregistrement lexical que l'analogie agit. Or les unités syntaxiques et sémantiques n'ont pas d'existence lexicale et fonctionnent indépendamment de la mémoire ${ }^{21}$ : ni les phrases ni leur sens construit ne sont enregistrés dans le lexique. Par conséquent, la construction d'une catégorie syntaxique ou sémantique ne pourra jamais être bloquée parce que le locuteur ne se souvient pas du bon résultat, ou parce que celui-ci n'est pas enregistré dans le lexique. Le «bon» résultat, ici, est

20. Joaquim Brandão de Carvalho, dans sa contribution, soutient qu'il existe une forme d'analogie qui agit en dehors de toute référence lexicale. Il ne sera donc pas d'accord avec ce que je dis ici et fait, me semble-t-il, la prédiction que la syntaxe et la sémantique aussi devraient être touchées par l'analogie.

21. Pour ce qui est de la sémantique, les Signifiés sont bien sûr enregistrés avec les Signifiants. Mais le sens d'une phrase auquel ils contribuent n'est pas enregistré dans le lexique. Par ailleurs, on voit mal comment, lorsqu'un locuteur a oublié le sens d'un mot, il s'en fabriquerait un à partir d'autres sens (et sans impliquer le Signifiant). 
toujours acquis par voie grammaticale et in vivo; il n'est jamais affaire de lexique.

En somme, donc, l'absence de conditionnement analogique en syntaxe et en sémantique n'est qu'une conséquence, par ricochet, de la particularité phonologique traitée en section 2: la phonologie (en compagnie de la morphologie dérivationnelle) est la seule discipline à devoir départager les facteurs lexicaux des produits grammaticaux; c'est pour cette raison qu'elle est soumise à l'analogie, et que la syntaxe et la sémantique, vierges de conditionnements lexicaux, y échappent.

\section{Contrairement à la syntaxe, le nombre d'objets bien formés en phonologie est fini : les dictionnaires existent}

4.1. Un constat a priori accablant: le nombre d'unités produites par la phonologie est fini, et la récursivité inconnue

La première phrase qu'un étudiant entend dans un cours d'introduction à la grammaire générative est la suivante: «l'objectif du linguiste est de construire un mécanisme qui génère toutes les phrases bien formées de la langue, et seulement celles-ci ». La seconde fait observer que ce mécanisme ne saurait être la simple mémorisation de la liste des phrases bien formées, car le nombre de celles-ci est infini. La raison en est la récursivité : on peut enchâsser des phrases, à l'infini. Ce qui s'y oppose au bout de trois ou quatre subordonnées n'est pas la grammaire, mais des facteurs extralinguistiques telle la mémoire.

Poursuivons : un locuteur ne peut mémoriser toutes les phrases bien formées car la capacité de mémoire de l'homme est certes grande, mais elle n'est pas infinie. Il faut donc se rendre à l'évidence que l'humain se sert, lorsqu'il produit de la parole, d'un ensemble statique d'éléments dans lequel il puise, et d'un mécanisme concaténatif qui assemble les pièces détachées. C'est ce que l'on appelle le lexique et la grammaire, et c'est la dernière qui fait, entre autres choses, que l'homme est homme : lui seul la possède (c'est la seconde articulation martinetienne).

Noam Chomsky est un syntacticien (bien qu'il ne l'ait pas toujours été), et le raisonnement qui vient d'être exposé, 
sans surprise, est syntaxique. Car c'est la syntaxe que les étudiants rencontrent d'abord (d'autres temps ont connu un autre parcours) et qui sans doute leur parle le plus. Or, tout est faux dans ce raisonnement lorsque l'on tente de l'appliquer à la phonologie. Celle-ci en effet possède des propriétés exactement inverses: le nombre des expressions phonologiques bien formées est parfaitement fini, et la récursivité inconnue.

On a depuis toujours fabriqué des dictionnaires, i.e. des listes de mots. Ceux-ci sont plus ou moins complets, mais il ne fait aucun doute que les mots d'une langue sont en nombre fini (même en faisant la part belle aux néologismes). Si on considère que les mots sont des expressions phonologiques, alors celles-ci, contrairement à leurs cousins syntaxiques, peuvent être constituées en liste.

On peut certes chercher d'autres expressions phonologiques : si le lexique de la syntaxe sont les mots et son produit, des phrases, les blocs de construction de base de la phonologie sont les phonèmes, et le résultat de leur concaténation, des mots. Or il est encore beaucoup plus facile de dresser l'inventaire exhaustif des phonèmes d'une langue qu'il ne l'est de confectionner un dictionnaire. Aussi, la liste des phonèmes va être beaucoup plus réduite, une vingtaine d'unités pour beaucoup de langues, une cinquantaine pour les systèmes vraiment complexes.

Ensuite, la récursivité n'a pas cours en phonologie, ni d'ailleurs dans l'autre module interprétatif des structures syntaxiques, la sémantique. C'est la raison pour laquelle Chomsky fait maintenant la différence entre LFN, Language Faculty in the Narrow Sense, et LFB, Language Faculty in the Broad Sense (Chomsky et al. 2002). Le contenu par excellence de la Grammaire Universelle (mais non le seul) serait la récursivité, propre à la syntaxe et à elle seule ${ }^{22}$. Il existe des

22. Dans le modèle minimaliste, la récursivité est le produit de l'opération Merge ( $c f$. ci-dessous). La FLN serait donc faite de celle-ci, ainsi que de la capacité à communiquer avec les modules interprétatifs, la sémantique et la phonologie. Cette faculté est appelée Phase (Chomsky 2001). La syntaxe est donc le seul module à être caractérisé par la FLN, qui est faite de Merge et de Phase. 
Présentation. En quoi la phonologie est vraiment différente

propriétés universelles sémantiques et phonologiques, mais elles sont d'un autre ordre.

L'absence de la récursivité en phonologie, dont Chomsky ne fait que prendre acte, a des conséquences disciplinaires importantes.

\subsection{Conséquences pour la théorie phonologique}

D'abord pour la théorie phonologique elle-même ${ }^{23}$. S'il n'y a pas de récursivité en phonologie, le module correspondant doit être incapable d'en produire. Or dans le paysage minimaliste (Chomsky 1995), l'opération Merge est responsable de la construction arborescente : elle colle un nœud branchant (branchant une seule fois, bien sûr) ou une de ses jambes à un autre nœud. Or la récursivité, formellement, est définie comme la domination d'un nœud d'une certaine catégorie par un nœud de la même catégorie (p.ex. un génitif: un groupe nominal en spécifie un autre). Par conséquent, Merge est l'architecte de la récursivité. Il n'y a pas de récursivité sans Merge. Et donc, à l'inverse, l'absence de récursivité implique l'absence de Merge: il n'y a pas de structure arborescente possible qui soit nonrécursive. On en conclut, donc, que Merge ou bien est à l'œuvre et alors produit et arborescence et récursivité, ou bien est absent, auquel cas le résultat ne présente ni l'une ni l'autre structure.

Toute théorie phonologique est donc invitée à être incapable de produire de la récursivité. Si une théorie devait proposer des structures arborescentes, il est exclu que celles-ci soient dues à Merge ou à une opération équivalente. Or l'existence de structures arborescentes construites par Merge en syntaxe et par une autre opération en phonologie poserait le problème de l'homogénéité de la grammaire : est-il raisonnable de supposer que les mêmes structures exprimant les mêmes rapports hiérarchiques et servant le même propos naissent de deux manières différentes? Comme toutes les théories phonologiques, depuis l'introduction de l'idée autosegmentale, regorgent de structures arborescentes, la question à propos de

23. Ce qui suit ici est un résumé de l'introduction générale de Scheer (2004: $\S 2)$. 
leur naissance et, comparativement, de celle de leurs cousins syntaxiques, est assez brûlante ${ }^{24}$.

Il est une théorie phonologique, cependant, dont le résultat est une structure "plate", i.e. qui ne comporte pas d'arborescence du tout. Il s'agit de "CVCV », la théorie dans laquelle je suis engagé (Lowenstamm 1996, Scheer 1999, 2004, Szigetvári 1999). Il faut bien noter que son caractère plat n'est pas l'objectif de la démarche qui la motive, mais bien son résultat. La motivation est ailleurs et n'a aucun rapport avec la récursivité. L'idée fondatrice de la Phonologie de Gouvernement dont est issu CVCV, la seule en réalité, est la latéralité : les relations entre les segments qui structurent la chaîne parlée (notamment en unités syllabiques) ne sont pas de nature arborescente, mais latérale. Les segments communiquent directement les uns avec les autres (Gouvernement et Licenciement) et ne prennent pas le détour de nœuds arborescents qui se trouvent au-dessus d'eux. Les noyaux vides, qui sont le signe de marque de la Phonologie de Gouvernement, doivent leur existence à ces relations latérales (et non l'inverse).

L'absence de structures arborescentes est donc le produit nécessaire du programme latéral. Au prime abord, celuici paraît difficilement défendable car chacun sait que la linguistique est affaire de hiérarchie, et donc d'arborescence. Etant donné le leadership de la syntaxe, la phonologie générative a toujours cherché à imiter les structures proposées en syntaxe. Comment peut-on donc croire en une théorie phonologique plate si la syntaxe est profondément arborescente?

En prenant acte de la différence majeure entre la syntaxe et les autres modules de la grammaire - la récursivité -, une phonologie plate non seulement perd tout son exotisme, mais encore explique cet état de fait plutôt que de se contenter de le constater. Car s'il n'y a pas d'arborescence, il ne peut y

24. Elle n'est pourtant pas à l'ordre du jour de la discussion phonologique contemporaine, hélas, ce qui témoigne de l'éloignement de plus en plus marqué, théorique, institutionnel, intellectuel, pratique et personnel, de la syntaxe et de la phonologie depuis les années 80 . Nul ne peut douter que celui-ci est néfaste pour la linguistique entière. 
avoir de récursivité : j'ai montré supra que la seconde suppose nécessairement la première. Et donc, en parfait accord avec Chomsky et al. (2002), la phonologie (au même titre que la sémantique) est un monde sans Merge.

Il est utile, ici encore, d'insister sur le caractère non circulaire du raisonnement : la Phonologie de Gouvernement n'a jamais cherché à expliquer l'absence de récursivité en phonologie. Pour des raisons qui relèvent de l'organisation phonologique interne et d'elle seule, elle a mis en œuvre le programme latéral - dont un résultat secondaire en effet est l'absence d'arborescence et, partant, l'inexistence de Merge et l'impossibilité de récursivité.

4.3. Conséquences pour l'architecture générale: les arguments génératifs classiques contre le behaviourisme n'ont pas de mordant en phonologie

La théorie générative est née, dans les années 50 , de l'opposition à l'empirisme en général, et à son incarnation en psychologie, le behaviourisme, en particulier (Chomsky 1959).

En philosophie, l'empirisme est représenté par Aristote dans l'antiquité et, depuis le Moyen-Age, se manifeste typiquement dans le domaine anglo-saxon (David Hume et John Locke au $18^{\text {ème }}$ siècle œuvrant contre les philosophes « spéculatifs », Bertrand Russel au $20^{\text {ème }}$ siècle). L'humain ici, à sa naissance, est une « feuille blanche » (Locke). A partir de là, aucun énoncé qui ne soit basé sur des faits empiriques ne peut être admis, même sous forme d'hypothèse ; le positivisme, autre incarnation de l'empirisme, enseigne que la méthode en sciences est inductive et seulement inductive (bottom-up) : des données sont reliées à d'autres données, et de ce réseau émergera inéluctablement la vérité (Rudolf Carnap, Ludwig Wittgenstein); seule l'observation fréquente et répétée mène à la compréhension de la nature ; un énoncé est vrai lorsqu'il a été vérifié expérimentalement (et seulement dans ce cas).

A cette vision du monde s'oppose le mentalisme. Platon est souvent donné comme le représentant de ce courant dans l'antiquité ; Descartes, Kant, von Humboldt ou encore Popper, avec la concentration cartésienne sur l'aspect cognitif que l'on appelle rationalisme, l'incarnent plus récemment. Ici, l'humain 
est autre chose qu'une simple machine perfectionnée. Son développement est guidé par toute sorte d'influences parmi lesquelles l'observation et la répétition, certes, mais qui assurément ne sont pas seules déterminantes : elles restent vaines et sans résultat en l'absence du génie propre à l'humain (et à lui seul), qui structure et valide les expériences sensorielles. En ce qui concerne la méthode en sciences, ici, la compréhension du monde peut procéder de n'importe quelle attitude et être acquise par quelque méthode que ce soit, y compris par un raisonnement qui n'a aucun fondement sensoriel, empirique ou expérimental.

En linguistique, l'opposition entre empirisme et mentalisme (ou rationalisme) a son expression la plus saillante lorsque la question est posée de savoir comment l'enfant vient à maîtriser sa langue maternelle. Selon les empiristes qui en psychologie s'appellent behaviouristes (Skinner 1957), l'acquisition du langage est le résultat exclusif de l'interaction continue entre un stimulus et une réponse. L'enfant cherche à imiter les adultes et ainsi se rapproche petit à petit de sa cible. Dans cette perspective, il apprendra sa langue maternelle par le même procédé qu'il utilisera plus tard pour apprendre à jouer du piano, ou à conduire une voiture: il observe, essaie d'imiter, corrige son tir et répète ce mouvement jusqu'à ce qu'il arrive à la perfection.

L'acquisition du langage ayant été classiquement une branche de la psychologie et non de la linguistique, c'est la théorie psychologique dominante de l'après-guerre, le behaviourisme, qui était également celle de l'acquisition. Chomsky (1959) la conteste: la langage n'est pas un objet comme un autre. Sauf défaut cérébral physiologique ou absence de congénère pendant la période critique ${ }^{25}$, il est une propriété

25. Un enfant dépourvu de compagnie humaine ne parlera pas : les divers cas d' « enfants sauvages» (ou «enfants-loups») qui ont été recensés le confirment. En revanche, démonstration a été faite que deux petits humains n'ont besoin d'aucun stimulus linguistique afin d'acquérir le langage, qui émergera "spontanément»: Kegl et al. (1999) documentent un cas où deux enfants sourds ont développé, en l'absence complète d'une quelconque instruction, un langage gestuel présentant toutes les caractéristiques propres au langage humain. 
humaine inaliénable qui définit l'espèce. Car il n'y a que l'homme qui parle, et il n'y en ait pas qui ne parle. Apprendre à parler et à conduire une voiture sont donc deux activités différentes par principe : l'une est pratiquée par tout humain, l'autre par une petite minorité seulement. Et, surtout, l'une est régie par des conventions que l'homme a établies (p. ex. le code de la route), alors que l'autre est un système que l'homme n'a pas créé et sur lequel il n'a pas d'influence (la grammaire). Le langage n'est pas un objet conventionnel ou artefactuel; produit de l'évolution, c'est un objet naturel au même titre que le foie, un atome ou une association d'hydrocarbure. Par conséquent, ce sont des lois naturelles indépendantes de la volonté de l'homme qui le régulent et président à son destin. Enfin, le procédé qui permet à l'homme de l'acquérir est fondamentalement différent de celui qui est utilisé afin de s'approprier d'autres compétences cognitives très sophistiquées (conventionnelles ou non), qui, elles, doivent « s'apprendre ».

L'opposition entre l'acquisition du langage et toute autre chose que l'homme apprend est classiquement basée, dans la littérature générative, sur trois observations.

D'abord les propriétés même du processus acquisitionnel et de son résultat. Les humains apprennent plus ou moins bien à conduire une voiture, à jouer du piano ou à manipuler un traitement de texte informatisé. Le langage ne fonctionne pas ainsi : le succès de l'apprentissage est toujours complet et total; il ne dépend en rien de la motivation ou l'intelligence du sujet ${ }^{26}$. A la fin du processus d'acquisition, tout homme sait parfaitement sa langue (sauf défaut cérébral), et il n'existe pas de hiérarchie entre ceux qui sauraient plus ou moins bien parler que d'autres (sauf bien sûr dans un sens sociologique qui est sans intérêt ici).

Ensuite, l'acquisition du langage n'est pas quelque chose que l'homme décide : il ne peut s'y soustraire. Il pourra autant qu'il voudra décider de ne pas l'acquérir, ou d'ailleurs de l'oublier, cela n'aura aucun effet: l'homme est condamné à

26. Dans les limites, bien sûr, des sujets « normaux », où la « normalité » n'est pas linguistiquement définie. 
parler. Rien de tel, bien sûr, du côté de l'apprentissage d'activités conventionnelles.

Enfin, le processus de l'acquisition est entièrement indolore, à tel point que les sujets ne s'aperçoivent même pas qu'ils sont en train d'accomplir un travail d'Hercule. On n'a jamais vu un enfant suer à cause de l'effort qu'il consent lors de l'acquisition, ni de se plaindre de sa difficulté. C'est encore ici un contraste évident avec tout autre apprentissage qu'on demande à un jeune humain (piano, écriture etc.). Au contraire, on retrouve ces mêmes caractéristiques, point par point, lors de l'apprentissage de compétences génétiquement conditionnées et inaliénables à l'homme telles que marcher ou manger.

Le second argument est connu sous l'étiquette pauvreté $d u$ stimulus. Il est un fait que les locuteurs n'ont jamais entendu la grande majorité des phrases auxquelles ils sont exposés tous les jours, et que celles-ci même n'ont jamais été prononcées par quiconque. Pour autant, ni celui qui les prononce ni celui qui les entend n'éprouve la moindre difficulté à les utiliser, sans aucun risque de malentendu. En outre, tous les locuteurs d'une langue partagent le même jugement de grammaticalité, positif ou négatif, sur les phrases qu'on leur propose (sauf éventuellement des zones d'ombre aux marges de la grammaticalité), y compris si celles-ci sont inconnues. Ils ne se sont pourtant pas donné le mot, et nul ne leur a enseigné comment distinguer les bonnes des mauvaises phrases. C'est leur compétence (leur Langue) qui leur permet de faire le tri. Or celle-ci n'a pu être acquise en imitant un comportement car les sujets n'y ont jamais été exposés.

Il n'est pas clair comment fonctionne ce que l'on appelle « inférence», et qui est régulièrement mis en avant par les behaviouristes. Quels qu'en soient le fonctionnement et la réalité, les informations dont un enfant dispose ne lui permettent certainement pas de conclure à tout ce qu'il sait de sa langue. C'est notamment vrai pour le savoir négatif : comment un enfant inférerait-il, par exemple, que toute phrase où un pronom est lié dans un domaine local est agrammaticale (liage)? Il a été confronté aux seules phrases où le pronom est libre, et nul ne lui a dit qu'il faut qu'il le soit. C'est ce que Chomsky appelle « knowledge without grounds ». La conclusion en est que l'enfant sait déjà un certain nombre de choses avant qu'il ne soit exposé à 
la moindre donnée linguistique et ne puisse imiter quoi que ce soit. Ce savoir est consigné dans la Grammaire Universelle, et transmis par voie génétique.

Enfin, les universaux constituent le troisième argument. S'il était vrai que l'enfant ne fait que répéter ce qu'il entend, il devrait être capable d'acquérir n'importe quel système et son contraire, de manière aléatoire. Or il est un fait qu'une langue naturelle, que le langage humain tout court, est tout sauf une mosaïque de propriétés arbitraires. Une réglementation assez sévère définit ce qu'est une langue naturelle possible. C'est ce que le modèle génératif appelle la Grammaire Universelle. Parmi les exemples de ce qui gendarme la variation d'une langue se trouvent les principes de liage qui ont déjà été mentionnés : ils sont parfaitement universels et prédisent qu'aucune langue naturelle n'a pu, ne peut et ne pourra avoir un système où les pronoms sont liés, ou les anaphores libres, dans leur domaine local.

La réponse des behaviouristes, bien sûr, est que les universaux existent justement parce que les enfants n'ont jamais l'occasion d'entendre autre chose. S'ils étaient exposés à une langue où les principes de liage marchent sur la tête, ils l'acquerraient autant que n'importe quelle langue où ils sont à l'endroit. Mais ceci ne fait que déplacer le débat en diachronie : si tel était le cas, comment se fait-il que les enfants ne soient jamais exposés à un stimulus qui contrevient aux universaux ? En d'autres termes, pourquoi est-ce que les langues n'évoluent pas de manière anarchique? Pourquoi y a-t-il certains changements récurrents, alors que d'autres ne se produisent jamais ? La réponse générative est toujours la même : parce que toute production langagière humaine, synchronique ou diachronique, est sous haute surveillance de la part de la Grammaire Universelle. Un changement qui serait contraire à ses dispositions ne peut avoir lieu. Les behaviouristes, quant à eux, n'ont pas de réponse à cette question : les langues devraient pouvoir évoluer dans tous les sens.

Considérons maintenant la situation de la phonologie par rapport à ces arguments. Il n'y a que le premier, celui concernant l'aisance et le succès garanti de l'acquisition, qui y vaille. Le second, la pauvreté du stimulus, n'est pas applicable en phonologie à cause notamment du nombre fini des 
expressions phonologiques bien formées. Celui-ci est lui-même la conséquence de l'absence de récursivité en phonologie.

On retombe ici sur l'enracinement lexical de la phonologie qui a déjà été déterminant lors de la discussion de la diachronie et de l'analogie plus haut. L'espace dans lequel se déroule la phonologie n'est pas la phrase, mais le mot. Or les mots sont enregistrés dans le lexique et donc en nombre fini. Qui plus est, il faut avoir appris un mot pour le connaitre et pouvoir le juger - contrairement à une phrase, qu'on n'apprend pas par cœur ni n'a besoin d'avoir entendue pour en avoir une opinion en termes de grammaticalité. Si un locuteur n'a jamais entendu une phrase grammaticale, il va sans hésiter la déclarer bien formée. Si en revanche on présente à un locuteur un mot bien formé qu'il n'a jamais rencontré, son jugement va être beaucoup moins affirmatif, sinon nul.

Or le fonctionnement des mots est de l'eau sur le moulin behaviouriste : certes les mots ne sont pas uniquement affaire de mémoire, mais ils sont aussi affaire de mémoire. Il ne peut y avoir de mot sans stimulus préalable. La maxime behaviouriste semble donc être juste ici : "rien n'existe dans le cerveau qui n'a préalablement existé dans les sens ».

Autrement dit, il n'y a des lacunes distributionnelles accidentelles qu'en phonologie (et en morphologie). La syntaxe n'en connait pas : toute phrase possible est une phrase existante. Alors que tout mot (ou tout morphème) possible n'est pas nécessairement un mot (ou un morphème) existant. Certes les locuteurs ont une intuition sur ce qui est un mot possible et ce qui ne l'est pas (sur cette notion, $c f$. Mohanan 1986 : 52ssq). Par exemple, plite est certainement possible en français, mais n'existe pas. Si demain quelqu'un y attache un Signifié et que cette association fasse fortune, un nouveau mot sera né. La même chose n'est pourtant pas vraie pour lpite: on pourra y associer autant de Signifiés que l'on voudra, son groupe initial le disqualifie définitivement. Tous les francophones conviendront du statut différent des deux séquences.

Le pouvoir générateur en phonologie, quoique gendarmé par le lexique et produisant seulement un nombre fini d'objets, n'est donc pas nul. Mais il est sans commune mesure 
avec celui de la syntaxe, ce qui fait qu'il ne peut guère servir d'argument dans la discussion sur la pauvreté du stimulus. ${ }^{27}$

Enfin, le troisième argument, les universaux, en principe est applicable à la phonologie : il n'y a pas de raison justifiant qu'aucune langue n'évolue en installant un abrègement en syllabe ouverte, un allongement en syllabe fermée, une palatalisation devant coup de glotte ou quelque autre monstre phonologique.

Mais il y a ici une parade behaviouriste qui n'est disponible qu'en phonologie: le phonétisme dont il a été question supra. Dans cette perspective, le changement diachronique a pour seule et unique source des erreurs de perception faites par les enfants. L'évolution est donc conditionnée par des propriétés phonétiques, et uniquement par celles-ci. Cette position est défendue, entre autres, par Hale \& Reiss (2000) et Blevins (2004). Hale \& Reiss pour autant n'abandonnent pas tout à la phonétique: le niveau nonsubstantiel (i.e. non-mélodique, telle la structure syllabique) relève selon eux de la phonologie autonome. Mais l'Inductive Grounding (cf. note 12, dont Blevins 2004 est une incarnation), qui emporte très largement l'adhésion au sein de la théorie de l'optimalité, relève du phonétisme le plus complet, soutenant que tout phénomène phonologique, $\mathrm{y}$ compris les effets syllabiques, ont une cause non-phonologique.

Dans ces conditions en effet, rien n'empêche de voir dans l'invariabilité du changement diachronique le simple reflet de la phonétique qui est universelle par définition (l'anatomie buccale, l'aérodynamique, le système auditif, etc. sont partagés par tous les humains).

La phonologie est donc encore ici différente, cette fois à cause de son interface avec la phonétique : nul ne prétendra que

27. Wauquier (en préparation) au contraire soutient que si le stimulus phonétique est riche, le stimulus phonologique est aussi pauvre qu'en syntaxe: la richesse phonétique ne fournit aux enfants aucun mode d'emploi qui leur révélerait comment le signal continu peut être ramené à des unités phonologiques discrètes. La gestion des performances moteurs, etc. peut se faire sur la seule foi de la phonétique jusqu'au moment où les enfants manipulent des unités significatives - des mots -, dont l'existence suppose l'opposition et, partant, le phonème. 
l'évolution syntaxique ou sémantique produit certains changements et en exclut d'autres parce qu'elle y est contrainte par la phonétique. La validité de l'argument construit sur la foi des universaux, en phonologie mais non en syntaxe ni en sémantique, dépend du débat plus général concernant le phonétisme: existe-t-il une phonologie immaculée (compétence, Langue) ou non ? Si la réponse est oui, la parade behaviouriste est ratée; si c'est non, elle est de nature à rendre l'argument des universaux inapplicable en phonologie.

En somme, donc, les arguments en faveur d'une Grammaire Universelle génétiquement transmise qui sont présentés dans les manuels, dans les cours d'introduction et même dans la littérature spécialisée sont justes - en syntaxe. On oublie systématiquement de mentionner que la situation est quelque peu différente en phonologie. Un exemple parlant de ce syntactico-centrisme, qui du reste est coutumier dans le milieu génératif, est un numéro spécial de la revue The Linguistic Review $\left(\mathrm{n}^{\circ} 19,2002\right)$ consacré entièrement à la pauvreté du stimulus : on y trouve neuf articles, dont aucun ne discute la situation en phonologie.

Enfin, on s'en doute, les neo-behaviouristes choisissent comme porte d'entrée dans l'édifice de la grammaire autonome la phonologie (p. ex. Carr 2000, 2003), qui est beaucoup moins armée que la syntaxe ou la sémantique et représente donc une victime plus facile. Aussi, l'éloignement de la syntaxe et de la sémantique d'un côté, et de la phonologie de l'autre, qui va croissant depuis les années 80 , a d'étranges conséquences qui ne sont pas communément perçues: on ne rencontre guère d'attitude ou d'analyse behaviouriste en acquisition de la syntaxe, alors que celles-ci foisonnent, le plus souvent nonavouées et même non-assumées, en acquisition de la phonologie.

4.4. Conséquences pour la méthodologie : la phonologie peut, la phonologie doit utiliser des corpus

Qui dit liste finie dit, bien sûr, corpus. Aucun syntacticien ne pourra jamais raisonner sur la totalité des observables qui constituent son objet d'étude. C'est vrai, aussi, 
pour la sémantique, je pense, car il y a autant de sens différents, potentiellement, qu'il y a de phrases. Le phonologue, en revanche, peut espérer travailler sur des données exhaustives. Pour lui, l'exhaustivité n'est pas à jamais hors de portée; elle est une simple question de faisabilité technique.

Et dans ce domaine, l'évolution des dix dernières années a créé un tout nouvel horizon. Le matériau de base du phonologue sont les mots, et les mots, depuis toujours, sont inventoriés dans des dictionnaires. Les phonologues ont toujours utilisé les dictionnaires, mais ne pouvaient s'en servir vraiment que lorsqu'ils recherchaient des propriétés du début de mot. En complément, il y avait quelques dictionnaires inverses, souvent artisanaux et de volume, portée et qualité fort variables. Typiquement, le phonologue se promenait avec un calepin et notait, tous les jours, les mots que le vent lui apportait. Si par exemple, en travaillant sur le schwa, on voulait savoir combien de mots en français présentent une séquence occlusive-schwaobstruante-liquide, on restait bredouille, à moins de rencontrer François Dell qui avait systématiquement inventorié les mots à schwa depuis des années. La réponse, dans ce cas, est qu'il existe un seul mot de ce type : degré.

Les moyens techniques, depuis, ont fait des progrès dans deux domaines. D'une part les dictionnaires ont été informatisés et existent maintenant sous forme électronique ${ }^{28}$. D'autre part, les moyens d'interrogation sont tels qu'en principe n'importe quelle requête peut être satisfaite instantanément en appuyant sur un bouton. En principe - mais souvent la réalité est assez différente, laissant le phonologue bredouille comme auparavant. C'est que d'une part les dictionnaires électroniques ne sont pas conçus pour l'usage des linguistes et n'admettent pas, sous le logiciel original, les recherches dont le phonologue a besoin. D'autre part, les éditeurs électroniques cherchent à " protéger» leurs données en ne donnant pas accès à la liste complète des entrées, qu'il est impossible d'exporter. On est alors contraint à « craquer » le code du CD afin de s'approprier

28. C'est vrai au moins pour les «grandes» langues occidentales; bien d'autres sont encore loin, très loin parfois, du compte, $c f$. mon propre article dans ce volume. 
son contenu sous une forme neutre et exploitable aux fins de la recherche par un logiciel standard.

Par ailleurs, il existe des limitations liées à la structure de la base de données elle-même. Il est évident que les éditeurs n'ont pas construit leur dictionnaire en fonction des besoins de recherche des phonologues. Si une recherche fait appel à une propriété secondaire des mots telle leur étymologie ( $c f$. l'article de Joaquim Brandão de Carvalho) ou leur relation avec d'autres mots (par exemple les paires itératif - non-intératif du même mot, $c f$. mon propre article), c'est la loterie. Cet état de fait peut conduire les phonologues à construire leur propre base de données, renseignée certes par des dictionnaires papier existants, mais en recoupant et en regroupant des informations en provenance de plusieurs sources lexicographiques, et en fonction du projet de recherche particulier. Un exemple de cette pratique est le récit détaillé concernant le somali que Xavier Barillot présente dans sa contribution au présent volume.

Cette discussion sur les dictionnaires électroniques suppose tacitement que la première ressource des phonologues est, justement, le dictionnaire ou, plus généralement parlant, les corpus lexicographiques. Je pense que c'est vrai - mais bien sûr ce n'est pas toute la vérité. Il existe des informations pertinentes pour le phonologue qui par la nature de l'objet ne sont pas contenues dans les dictionnaires, qu'ils soient électroniques ou classiques. Il s'agit d'abord de tous les mots qui n'ont pas (encore) acquis assez de noblesse pour figurer dans les dictionnaires, ensuite des acronymes, de l'argot ou encore des langages secrets ou ludiques de type verlan qui en général ne font l'objet d'aucun recensement lexicographique ( $c f$. les articles d'Olivier Rizzolo et de Claudine Pagliano).

C'est ici que les corpus textuels font leur entrée en scène. Les mots qui n'ont pas (encore) le droit de cité dans les dictionnaires pourront se trouver au milieu d'un texte, qui peut être d'origine diverse et avoir des propriétés variées: corpus oraux, corpus recueillis de manière dirigée ou semi-dirigée ( $c f$. le PFC dont il est question dans l'article de Jacques Durand et Julien Eychenne), corpus littéraires, journalistiques ou politiques (ces trois catégories de textes sont le plus facilement accessibles sous forme électronique), ou encore corpus «nationaux» dont la 
vocation est de compiler toute production langagière pertinente afin de fournir une « image moyenne » de la langue en question.

Parmi toutes ces ressources, il en est une qui se recommande par son étendue inégalable, ainsi que par son accessibilité totale et permanente : Internet. On voit de plus en plus fréquemment des phonologues, pour le besoin d'une évaluation «rapide» et "représentative» d'une fréquence lexicale ou du rapport numérique entre deux formes concurrentes, faire état d'une simple recherche Google. La stratégie est alors de faire précéder la présentation de ces données par des propos circonstanciés qui mettent en garde contre les éventuels écueils et les biais multiples qui amoindrissent leur validité. Il n'en reste pas moins que ces données sont ensuite utilisées dans un argumentaire scientifique, et mises en concurrence avec des données de source plus contrôlée. Un cas de mon propre vécu est Steriade (2003) qui, tout en présentant sa moisson Google, indique qu'elle s'est aperçue après coup que la moitié des occurrences du mot recherché avait comme provenance sa propre production scientifique, disponible en ligne et recensée automatiquement par Google.

Il convient donc d'être extrêmement précautionneux à l'égard de toute donnée en provenance d'Internet, surtout si elle a été acquise au moyen de moteurs de recherche de type Google. Plénat et al. (2002) expliquent les circonstances et les écueils de l'utilisation d'Internet en tant que ressource linguistique. L'inconvénient majeur en est l'impossibilité d'authentifier la source du texte : on ne peut savoir par exemple si l'auteur est un locuteur natif de la langue dans laquelle il s'exprime.

Les corpus textuels permettent aussi de contrôler, dans une certaine mesure, les conditions extérieures de la production des données : contexte linguistique, sociologique, institutionnel, etc. C'est une fonction que, bien sûr, les corpus lexicographiques ne peuvent remplir. Il en va de même pour tout ce qui a trait au sandhi, i.e. aux phénomènes qui se produisent à l'intersection entre deux mots. L'article de Jacques Durand et Julien Eychenne en présente une illustration. Il va sans dire qu'il faut ici étudier les mots en contexte, et que les corpus lexicographiques ne fourniront aucun renseignement utile. 


\section{T. SCHEER}

Il convient enfin de distinguer entre la constitution d'un corpus et son évaluation, ces activités étant toutes deux préalables à l'analyse phonologique elle-même. Les mots que l'on recueille par l'interrogation d'un corpus ne sont pas «les données ». Ce sont des candidats au statut de donnée. Les données n'existent pas dans l'absolu, et il est bien sûr naïf de croire, en science, qu'il est des données «objectives ». Toute donnée est nécessairement construite, et construite par un humain qui prend des décisions. La machine ne sert que d'outil d'aide à la décision ( $c f$. par exemple Dalbera 2002).

Il en découle que le linguiste a non seulement la prérogative de décider qu'il n'y a pas lieu de prendre en compte telle forme ou tel mot pour l'analyse - il en a l'obligation, et pour chacun des objets sur lequel portera son analyse. Le linguiste, au même titre que tous les autres scientifiques, est responsable de ses données. Il ne peut se prévaloir, après coup, d'excuses du type "c'est Internet qui a fourni cette donnée », "c'est marqué dans le dictionnaire », ou encore «je n'ai pas pu matériellement vérifier des milliers d'exemples ». Nul ne fournit des données sauf le linguiste. Le fait que souvent la taille des corpus ne permette plus la vérification manuelle de chacune des formes qui seront utilisées pour l'analyse ne change rien à cette responsabilité. Elle est engagée quoi qu'il arrive et ne saurait être évacuée par quelque propriété ou évolution technique que ce soit. Dans la pratique, cela implique aussi que les résultats des recherches au sein d'un corpus soient soumis au jugement de locuteurs.

Il est un dernier point qui mérite mention: tous les projets de recherche en phonologie ne se prêtent pas de manière égale à l'étude de corpus. Pour aller vite, les corpus ne sont d'aucun secours ni d'aucune utilité lorsque l'on étudie des processus dits post-lexicaux. Il s'agit là de la terminologie utilisée au sein une théorie phonologique particulière, la Phonologie Lexicale ( $c f$. supra), mais qui a acquis une valeur générale et fait maintenant partie de la lingua franca phonologique. Les alternances post-lexicales sont celles qui sont automatiques: elles ne sont conditionnées par aucune propriété morphologique ou lexicale, i.e. exactement ce que la Phonologie Naturelle Générative considérerait comme relevant 
d'une règle synchronique. A titre d'exemple, il est coutumier de citer l'aspiration en anglais, qui s'associe automatiquement à toute occlusive non-voisée qui se trouve à l'initiale de mot ou devant voyelle accentuée. Il n'est nul besoin d'étude particulière ici car on connaît le résultat par avance : aucune variation ne se présente. Or les corpus révèlent la variation ( $c f$. la contribution de M.-J. Dalbera-Stefanaggi et J.-Ph. Dalbera). Certes on peut essayer de montrer que ce qui était considéré comme un processus post-lexical en vérité est lexicalement conditionné. Il n'en reste pas moins qu'il est possible et nécessaire de faire le tri des projets de recherche : certains se prêtent à la confrontation avec les corpus, d'autres moins ou pas du tout.

Ce qu'il est utile de retenir au-delà des problèmes conceptuels et techniques liés aux corpus, c'est que les phonologues n'ont plus désormais le choix : ils doivent fonder leurs analyses sur des corpus qui donnent un aperçu numérique des forces lexicales en jeu. Cette obligation est la conséquence de la circonstance suivante, néfaste à bien des égards : la phonologie de par sa nature est irrémédiablement et intimement entrelacée avec le lexique, la diachronie et l'analogie ; elle a comme objet d'étude un ensemble fini d'unités. Cette spécificité se révèle donc être un avantage lorsque l'on se penche sur ses conséquences méthodologiques qui concernent, notamment, l'utilisation des corpus.

Relevant de la nature des disciplines linguistiques, elle conduit aussi, tout naturellement, à une différence méthodologique en regard de la syntaxe. Dans cette discipline, les corpus n'ont jamais été un moyen bien populaire ni répandu pour construire des données. Les syntacticiens, surtout génératifs, ont toujours préféré le travail avec les informateurs, ainsi que l'introspection. Les jugements de grammaticalité produits par les locuteurs natifs, plutôt que le verdict des corpus, sont ici la mesure des choses. C'est d'ailleurs ce choix méthodologique qui a été depuis toujours l'objet d'un discours justificatif de la part des générativistes (Chomsky 1965 : 19ssq), autant qu'il a été attaqué par ses adversaires (Hagège 1986: 56ssq). Il leur a valu le qualificatif de " linguistes en chambre ", ou encore de « science tour d'ivoire ». 
Quoi qu'il en soit, je pense qu'il y a un fond à ce débat, qui ne fait que refléter les propriétés fondamentalement différentes de la syntaxe et de la phonologie dans les domaines mentionnés. La syntaxe est en effet moins demandeuse de corpus, elle peut s'en passer souvent. En tout cas n'y a-t-il pas la même obligation, ni les mêmes possibilités qu'en phonologie. Ce n'est pas pour autant, bien entendu, qu'il faille s'interdire, en syntaxe ou en sémantique, de recourir aux corpus pour identifier des données intéressantes qu'il s'agit, par la suite, de travailler avec des informateurs selon la méthodologie classique basée sur les jugements de grammaticalité.

Il existe donc une inclinaison naturelle à utiliser les corpus en phonologie qui, avec le progrès des moyens techniques, se transforme en obligation. C'est par la nature des choses que la même inclinaison n'existe pas en syntaxe ${ }^{29}$.

\section{Seule la phonologie est structurale et structuraliste : les équivalents du phonème et du système phonématique sont inconnus en syntaxe et en sémantique}

\subsection{Le phonème et le raisonnement systémique sont absents} de la phonologie générative aussi parce que celle-ci s'inscrit dans une filiation syntaxique

Il existe, enfin, une autre spécificité de la phonologie qui est, je pense, de nature. Elle a eu des conséquences, importantes s'il en est, en conduisant à la structuration du $20^{\text {ème }}$ siècle en deux grands blocs théoriques : le structuralisme et le générativisme. Je veux parler du fait qu'il n'y a que la phonologie qui fasse système.

29. Le cas de la sémantique est plus complexe car il convient ici de faire la différence entre sémantique lexicale et non-lexicale. Il est bien évident que la dernière doit faire des études en contexte et donc travaille tout naturellement sur corpus - corpus textuel. Quant à la sémantique lexicale, l'utilité et la pratique des corpus dépend probablement du point de vue : les générativistes ne s'en servent guère, alors que leur usage au sein de l'école culiolienne est générale. 
L'idée structuraliste centrale, inaugurée en linguistique mais ayant rayonné bien au-delà de cette discipline ${ }^{30}$, est la suivante : un son n'est pas un objet linguistique en soi - il ne le devient que par les relations qu'il entretient avec d'autres sons du système auquel il participe. Autrement dit, il n'y a pas d'objet phonologique en isolation, en soi et pour soi; toute unité phonologique est nécessairement en relation avec d'autres unités du même type, et avec elles forme un système - une structure. D'où le nom de la théorie. Par conséquent, aucun objet phonologique ne peut être considéré seul : ni en tant qu'entité avec par exemple ses propriétés phonétiques individuelles, ni en tant qu'acteur d'un processus phonologique: deux objets phonétiquement identiques peuvent réagir au même processus de manière totalement différente en fonction de la place qu'ils occupent dans leurs systèmes respectifs.

Or le structuralisme est né en phonologie: Saussure, héritier (et acteur de premier plan : le mémoire) du $19^{\text {ème }}$ siècle et de l'école néogrammairienne (il fit ses études et passa sa thèse à Leipzig), est bien sûr phonologue, et le Cours ne propose que de la (morpho-)phonologie. Ensuite, à l'école de Prague où le phonème a été formellement inauguré (bien qu'il ait été une réalité déjà dans les analyses précédentes, y compris néo-grammairiennes), on faisait de la phonologie, pas de la syntaxe ou de la sémantique. Il en va de même pour la branche américaine du structuralisme qui est fondée sur la description phonologique - des langues amérindiennes. L'essor de la nouvelle perspective structuraliste après la guerre, en analyse synchronique autant qu'en diachronie, de part et d'autre de l'Atlantique, est une affaire de phonologues, et de phonologie. La syntaxe et la sémantique n'y ont guère contribué. Face au foisonnement phonologique, les travaux structuralistes en syntaxe sont peu nombreux, n'ont pas eu de postérité notable et du reste ne faisaient qu'appliquer la méthodologie développée en phonologie (Wells 1947 en est un exemple).

Je pense que l'occupation, au sein du structuralisme, de la quasi-totalité de l'espace par la phonologie n'est pas un

30. Par exemple en sociologie (Lévi-Strauss 1958, Bourdieu 1972), ou en psychanalyse (Lacan 1966) 
hasard. C'est au contraire la traduction fidèle d'une propriété naturelle qui oppose la phonologie aux autres disciplines linguistiques : le système. Les unités de base de la phonologie sont les phonèmes, et les phonèmes forment un système dont les membres réagiront collectivement si l'un d'entre eux est sujet, par un événement extérieur, à altération. Quelles sont les unités qui pourraient faire système en syntaxe ou en sémantique ? On $\mathrm{y}$ cherche en vain l'équivalent du phonème. Par conséquent, il n'y a pas de système non plus; les événements syntaxiques et sémantiques ne sont pas le résultat de pressions systémiques.

Il n'est pas surprenant, dans ces conditions, qu'une théorie fondée sur la syntaxe produise une phonologie qui ignore tout du système. Noam Chomsky a fait sa Maîtrise en phonologie (Chomsky 1951) et s'est ensuite intéressé à la syntaxe. Il inaugure le système procédural qui donnera son nom au futur modèle génératif dans sa Maîtrise, où des règles ordonnées relient une structure sous-jacente à une structure de surface $^{31}$. Par la suite, Chomsky applique ce système à la syntaxe dans Syntactic Structures (Chomsky 1957) et Aspects (Chomsky 1965). Dès lors, l'entreprise générative est fondamentalement syntaxique. On a d'ailleurs souvent reproché à sa vision globale un syntactico-centrisme à toute épreuve (p.ex. Jackendoff 1997, 2002); de même, son argumentaire philosophique est exclusivement syntaxique ( $c f$. section 4.3).

Il me semble donc que la phonologie générative qui naît au cours des années 60 et conduit, en 1968, à la publication de SPE, doit être considérée comme un enfant du programme syntaxique chomskyen, plutôt que comme un descendant de Chomsky (1951).

La filiation syntaxe $\rightarrow$ phonologie (au lieu de phonologie $\rightarrow$ phonologie) est une des raisons qui a fait que le phonème et par conséquent le système phonologique en tant que tel, ainsi que toute causalité systémique, sont absents de SPE et ensuite de la tradition phonologique générative. Pareille

31. La paternité des règles ordonnées en réalité semble revenir à Bloomfield (1939), mais on cite souvent le fait que Chomsky n'a pas eu connaissance de cet article en écrivant sa Maîtrise (Encrevé 1997, Koerner 2003). 
Présentation. En quoi la phonologie est vraiment différente

filiation ne peut permettre au système d'être transmis : il n'y a ni phonème ni système ni pression systémique (ou leurs équivalents) en syntaxe - indépendamment de toute considération théorique.

Certes ceci n'est pas la seule raison expliquant l'absence du phonème et de son apanage en phonologie générative. On se souvient de la démonstration par laquelle Halle (1959: 22sq) conclut à l'inexistence du phonème ${ }^{32}$. Celle-ci, dans la mémoire collective, a fait que la discipline entière a adopté l'attitude générative sans phonème. Anderson (2000) cependant doute que la valeur intrinsèque de cet argument soit pour beaucoup dans l'abandon du phonème. Quel que soit le rôle joué par l'assimilation russe, il est raisonnable de penser que celle-ci n'est pas la seule cause de l'absence du phonème en phonologie générative.

\subsection{La revanche du structuralisme}

Si la science doit être cumulative, et si nul n'a montré que l'idée structuraliste est sans fondement, on est en droit de considérer que le structuralisme a une revanche à prendre : la phonologie générative doit le réinstaller dans ses fonctions.

Or, depuis une trentaine d'années la phonologie générative a produit des analyses qui font l'exact contraire de l'enseignement structuraliste: on considère des sons, leurs propriétés phonétiques autant que leur comportement en tant qu'acteurs d'un processus, sans prendre en compte leurs propriétés systémiques.

C'est tout juste s'il y a à signaler, vers la fin des années 80 , une brève résurgence de l'idée structuraliste lorsque la sousspécification a été développée. Celle-ci propose que les segments ne sont pas spécifiés pour tous les traits dans le lexique ( $c f$. Steriade 1995 pour un survol). L'avantage en est le non-encombrement des représentations par les traits absents, ce

32. L'assimilation de voisement en russe affecte les phonèmes pour qui le voisement a une qualité distinctive autant que ceux qui n'ont pas de partenaire voisé (i.e. $/ \mathrm{ts}, \mathrm{t} f, \mathrm{x} /$ ). Ecrire une règle spécifique pour rendre compte de l'assimilation de voisement de ces derniers lorsqu'ils sont suivis d'une obstruante voisée encombre la grammaire inutilement car toutes les obstruantes, participant ou non à la corrélation de voisement, se font assimiler dans ce contexte. 
qui permet aux segments adjacents de communiquer à travers le segment sous-spécifié sans que les lignes d'association ne se croisent. Les valeurs lexicalement absentes sont installées par défaut à la fin de la dérivation. Se pose alors la question de savoir selon quel principe les traits sous-spécifiés sont sélectionnés. La sous-spécification contrastive a été une des solutions proposées : sont présents dans le lexique seulement les traits qui servent à distinguer deux phonèmes de la langue en question (Steriade 1987, Clements 1988 et Archangeli 1988). Les structures de ce type de théorie ne sont donc pas universelles car la sélection des traits sous-spécifiés est corrélée aux systèmes phonémiques particuliers.

Cet interlude mis à part, la phonologie générative a toujours, me semble-t-il, appliqué ce principe antistructuraliste : un son est un son quel que soit le système dans lequel il se trouve; il est convenu qu'il a les mêmes propriétés et le même comportement procédural partout au monde. Cette attitude, bien sûr, est aussi le résultat de la recherche des universaux, activité fondatrice du générativisme : la Grammaire Universelle étant en point de mire, on cherche à établir ce qui est stable et invariable à travers les langues.

Il a fallu attendre le milieu des années 90 pour assister à un retour de l'idée structuraliste dans les théories génératives. Mais, me semble-t-il encore ici, à un retour larvé et nonassumé : mis à part Clements $(2001,2002)$ dans une certaine mesure, je ne sache pas que quelqu'un ait fait le bilan, montrant que l'idée structuraliste a été absente du raisonnement génératif, et absente à tort, de même qu'il n'y a jamais eu aucun échange d'arguments de type "voilà pourquoi nous pensons que l'idée systémique n'est pas bonne ». Le phonème ainsi que ses vertus distinctives et systémiques ont fait leur entrée dans la théorie de l'optimalité lorsque celle-ci a cherché à se débarrasser des causes formelles en phonologie. Pour le mouvement des grounded constraints (Hayes 1999, Steriade 1997, Hayes et al. 2004, Beckman 1997), les événements phonologiques ont pour seule cause des facteurs externes: fonctionnels, phonétiques, psycholinguistiques, etc. Certes on fait ici référence aux travaux structuralistes, mais ne développe pas, autant que je puisse en 
juger, de raisonnement global sur l'évolution de la phonologie et l'absence de l'héritage structuraliste dans les travaux génératifs.

Il y a eu également, avant que la théorie de l'optimalité ne s'intéresse à la fonction distinctive des sons, une redécouverte de l'idée structuraliste au sein d'une autre théorie phonologique, la Phonologie de Gouvernement. Celle-ci a été tout aussi larvée que la précédente, et complètement indépendante d'elle. L'instrument théorique majeur que le groupe de recherche autour de Jonathan Kaye et Monique Charette à SOAS/ Londres a mis en place depuis 1993 sont les contraintes de licenciement (Licensing Constraints, cf. Kaye 2000,2001, Charette \& Göksel 1994, 1996, j'en ai fait un résumé dans Scheer 2003). L'idée est simple: des sons phonétiquement identiques peuvent avoir une identité phonologique différente en fonction du système dans lequel ils se trouvent. Leur identité est déterminée par les primitives mélodiques dont ils sont composés, et celles-ci ont besoin d'être licenciées les unes par les autres. Les primitives sont certes identiques partout au monde, mais leur capacité à licencier des pairs varie de système en système et ainsi dérive les inventaires phonématiques différents. Aussi, le comportement des primitives lors de processus phonologiques tels que l'harmonie vocalique est tributaire des mêmes contraintes de licenciement. En somme, donc, l'empreinte génétique d'un système particulier d'oppositions est déposée dans les contraintes de licenciement, qu'il faut acquérir afin de maîtriser le système. Ce qui est patent, encore et toujours dans cette affaire, c'est qu'à aucun moment, la littérature sur les contraintes de licenciement ne dit que ses propositions sont une application du programme structuraliste, ni ne mentionne seulement le mot «structuralisme ».

Le troisième cas récent où l'idée structuraliste est enfin introduite dans le raisonnement génératif sont les travaux de Nick Clements $(2001,2002)$. Je l'ai dit supra, la dimension historique du mouvement entrepris est ici pleinement assumée et exposée.

A tout prendre, donc, voici la conclusion que je ferai : si l'héritage structuraliste a été absent en phonologie générative pendant longtemps et quelles qu'en fussent les raisons, c'est un tort qu'il s'agit de réparer le plus rapidement possible, et de 
réparer en parfaite conscience historique. Nous faisons de la phonologie, et les forces systémiques, autant que les propriétés universelles du langage, agissent sur les segments en tant qu'objets individuels, ainsi que sur leur comportement lors de processus phonologiques. Aucune théorie ne peut faire abstraction de l'un ou l'autre conditionnement.

Or tout ou presque reste à faire: c'est à peine si les phonologues se rendent compte de la carence structuraliste dans le monde génératif, et s'ils en mesurent le caractère néfaste, ainsi que les conséquences. Encrevé (1997) attire l'attention sur cette question : la phonologie de l'après-guerre vit dans un imaginaire où la nouvelle théorie générative est censée être en rupture totale avec l'ancienne théorie structuraliste. Or la rupture est davantage sociale qu'elle n'est scientifique : il y a d'importants éléments de continuité entre l'ancien et le nouveau. La conscience de ce fait, en d'autres termes la démolition d'une doctrine sociale au profit d'un regard moins partisan, ne fait que naître.

\subsection{Corpus structuraliste vs. corpus générativiste}

Un aspect de la réhabilitation du structuralisme concerne directement l'objet de la revue Corpus : l'absence de raisonnement structuraliste en phonologie générative a fait que les corpus sont construits et utilisés, davantage aujourd'hui d'ailleurs qu'il y a vingt ou trente ans, dans la perspective crosslinguisitic. Celle-ci est une conséquence directe du générativisme car elle répond à la recherche des universaux, inspirée par l'idée de la Grammaire Universelle.

Il va de soi qu'ici comme ailleurs, les deux aspects systémique et universaliste produisent la phonologie. Il ne peut donc s'agir de discréditer la recherche des universaux. C'est la méthode qui est actuellement appliquée afin d'établir les universaux qui, je pense, est critiquable. Elle procède directement, me semble-t-il, de l'absence, dans l'univers génératif, d'un contre-poids systémique.

Voici en quoi il peut être utile d'opposer des corpus structuralistes à des corpus générativistes. Les premiers cherchent à tirer profit de l'étude d'un phénomène particulier dans une langue donnée en prenant en compte l'ensemble des 
propriétés de celle-ci : système phonématique, caractéristiques typologiques, processus à l'œuvre, états diachroniques antérieurs, systèmes voisins et évolution de la langue ${ }^{33}$.

Les seconds, à l'opposé, brassent un nombre impressionnant de langues qui se comptent souvent par centaines (format typique des thèses en phonologie produites outre-Atlantique depuis une dizaine d'années). Ils se caractérisent par le fait que le linguiste qui rassemble les données et les interprète ne connaît aucune des langues en question (ou alors la portion congrue), n'en a entendu aucune de ses propres oreilles et ne sait d'elles rien d'autre que les trois exemples qu'il a puisés dans une grammaire qu'il n'a pas lue entièrement et dont il ne sait pas évaluer la qualité. Des études de ce type sont fréquentes, produites surtout dans le cadre d'une thèse. On peut citer par exemple Kirchner (1998) (lénition), Gurevich (2004) (est-ce que la lénition crée ou annule du contraste phonologique ?), Zhang (2001) (tons de contour), Morelli (1999) (groupes d'obstruantes), Walker (1998) (harmonie nasale), Kaun (1995) (harmonie d'arrondissement) ou encore Casali (1996) (hiatus).

Fonder des raisonnements qui donnent lieu à des conclusions théoriques majeures sur quelques mots d'une langue dont on ne connaît rien et qu'on a repris de seconde ou troisième main rappelle une pratique de la phonologie générativie naissante. Un cas d'école ici est le Canadian Raising: l'ensemble de données connu sous ce nom a servi, dans un article important (Bromberger \& Halle 1989), à prouver que les règles phonologiques doivent être ordonnées. Le Canadian Raising repose sur la comparaison de deux dialectes, l'un étant une variété canadienne de l'anglais bien connue (dialecte A), l'autre un idiome voisin aux propriétés cruciales pour l'analyse (dialecte $\mathrm{B}$ ). Or en cherchant à localiser le dialecte $\mathrm{B}$, on s'aperçoit que la seule et unique source en est un article de trois pages écrit par Martin Joos (1942). Celui-ci dit avoir relevé les données décisives dans une classe en enseignant. Or les dialectologues de l'anglais canadien n'ont jamais pu retrouver

33. La BDLC et le THESOC, décrits dans la contribution de M.-J. DalberaStefanaggi et J.-Ph. Dalbera, représentent ce type de corpus. 
trace de ce dialecte $\mathrm{B}$, si bien que la question capitale des règles ordonnées et du dérivationnalisme tout entier a été débattue pendant des années sur la base de quelques mots de provenance franchement douteuse que personne n'a jamais pu vérifier. Kaye (1990) retrace le détail de cette anecdote, et on note que malgré cela le Canadian Raising est un fantôme qui encore aujourd'hui refait régulièrement surface lorsque la question dérivationnelle est traitée (par exemple à l'occasion du débat sur l'opacité au sein de la théorie de l'optimalité).

Je ne veux pas dire, bien sûr, qu'il est impossible de travailler sur des données d'une langue qu'on ne connaît pas. Mais je pense qu'il y a actuellement une tendance vers le crosslinguistic qui ne se recommande pas. Or cette orientation vers des corpus générativistes n'a pas toujours été celle de la phonologie générative. C'est ce que fait remarquer Gussmann (2003) : il fut un temps où d'importantes propositions théoriques étaient faites sur la base de corpus structuralistes, i.e. de monographies qui étudiaient en profondeur la phonologie d'une seule langue. A commencer, bien sûr, par The Sound Pattern of English lui-même, qui a été précédé par The Sound Pattern of Russian (Halle 1959) et une étude du tchèque (Kučera 1961), enfin suivi par des monographies sur le japonais (McCawley 1968), le français (Dell 1973) ou encore le polonais (Gussmann 1980, Rubach 1984).

Par ailleurs, il est vrai aussi qu'il y a des phénomènes en phonologie qui se prêtent plus ou moins bien à l'étude crosslinguistic. Mis à part les travaux pionniers de Greenberg (1966, 1978) dans un cadre purement descriptif sans orientation théorique particulière, un champ qui parmi les premiers a été labouré dans la perspective d'un corpus génératif est celui de l'accent. Bruce Hayes, d'abord dans sa thèse (Hayes 1981), ensuite dans un effort continu qui a abouti à Hayes (1995), a essayé de rassembler les différentes manières dont l'accent peut être distribué dans les langues du monde et, à partir de là, de déterminer ce qu'est un système accentuel possible. Les systèmes d'accent ont depuis fait l'objet d'autres études crosslinguistic, typiquement à l'occasion de thèses : Bailey (1995), Gordon (1999) (poids syllabique), de Lacy (2002) (accent conditionné par la sonorité des voyelles), McGarrity (2003) 
Présentation. En quoi la phonologie est vraiment différente

(accent primaire et secondaire). Etudier les systèmes accentuels à l'aide d'un corpus génératif est certainement moins risqué que d'essayer d'obtenir des informations sur un processus segmental comme par exemple la palatalisation.

Il n'en reste pas moins que la bonne méthode afin d'obtenir des renseignements typologiques ne me semble pas être celle où l'enquêteur est un spécialiste du phénomène étudié mais ne connaît rien aux langues qu'il exploite (top-down). C'est la situation inverse qui est à recommander (bottom-up) : celui qui fait l'étude se renseigne auprès de spécialistes de chaque langue ou chaque groupe de langues, et ainsi bâtit une pyramide en faisant des généralisations d'étage en étage. Cette méthode est certes moins commode, prend davantage de temps et ne permet pas de produire une thèse. Mais en alliant les compétences génératives (l'enquêteur) et structuralistes (les spécialistes des langues), elle est la seule à même de contrebalancer les effets néfastes du corpus génératif.

Il convient, enfin, de distinguer les problèmes liés à la constitution des corpus génératifs de leur valeur heuristique. Supposons que nous disposions, pour un phénomène donné, du corpus cross-linguistic parfait qui aurait pris en compte toutes les situations particulières de toutes les langues du monde et donne donc une photographie exacte de l'existant. Que nous apprendrait-il ?

Selon l'avis de Frederick Newmeyer, rien du tout. Dans un article intitulé "The irrelevance of typology for linguistic theory» (Newmeyer 1998), il rappelle que l'objectif de la linguistique n'est pas de caractériser ce qui est un système grammatical probable, mais ce qui est un système possible ${ }^{34}$. Par conséquent, les comptes effectués dans les corpus génératifs n'ont aucune valeur heuristique et ne promeuvent en rien la connaissance des propriétés de la Grammaire Universelle. Au contraire, ils l'entravent plutôt en suggérant une objectivité chiffrée qui en réalité est factice. Ceci d'autant que l'objet rare et

34. De même Lass (1984 : 278s) : «Overall I think it's a good idea for ALL markedness considerations to be excluded from phonological characterizations. [...] Segments ought to code only their own properties, not statistics of cross-language distribution ». 


\section{T. SCHEER}

marginal parfois révèle le fonctionnement d'un système entier, fonctionnement qui par ailleurs reste dans l'ombre. En dialectologie par exemple, c'est une expérience quotidienne : on trouve souvent, dans une aire marginale et insignifiante quant au nombre de locuteurs et à son étendue géographique, la clef qui seule permet de comprendre un grand nombre de systèmes voisins qui sont parfaitement «non-marqués ». La minorité, ici, « a raison », et la majorité, «tort». L'article de M.-J. DalberaStefanaggi et J.-Ph. Dalbera dans ce volume offre de multiples illustrations de cette situation.

Ceci transposé à l'échelle du système universel revient à reconnaître qu'une propriété présente dans une seule langue au monde peut être celle qui nous renseignera sur un aspect de la Grammaire Universelle. Or ceux qui pratiquent les corpus génératifs en général font l'inverse : si une propriété donnée est la portion congrue dans le concert des langues, alors elle est également négligeable pour la construction théorique. Si en revanche $90 \%$ des langues du monde présentent telle propriété ou tel processus, il doit s'agir là de quelque chose d'important que la théorie est appelée à prendre en compte, et ce de manière prioritaire. Cette attitude se traduit d'ordinaire par le fait que les objets non-marqués sont primitifs dans les théories, et les objets marqués, dérivés d'eux au moyen d'une opération supplémentaire.

Par exemple, c'est le cas d'un scénario populaire d'acquisition au sein de la théorie de l'optimalité : l'enfant naît avec une grammaire où toutes les contraintes de marque (markedness constraints) dominent toutes les contraintes de fidélité (faithfulness constraints) et donc ne produit que des objets maximalement non-marqués dans un premier temps (« tata » etc.). Le processus acquisitionnel vise alors à produire des objets marqués, et afin de ce faire, l'enfant agit : il doit promouvoir certaines contraintes de fidélité au-dessus de certaines contraintes de marque. Mais même en dehors de la question de l'acquisition, la marque se trouve au cœur de la théorie de l'optimalité : les contraintes sont formulées de façon à ce que toute la grammaire tende vers la production d'objets non-marqués (ONSET plutôt que NOONSET, NOCODA plutôt que CODA). 
Le point de vue de Newmeyer (1998) n'est pas infondé, mais je pense que les informations statistiques de type crosslinguistic, si elles reposent sur un corpus génératif qui est construit dans les règles de l'art structuralistes, ne sont pas dénuées d'intérêt pour autant. Si elles ne sont pas seules à définir ce qui est une donnée que la théorie doit prendre en compte, elles fournissent un outil au linguiste qui enrichit sa réflexion et peut l'aider à prendre des décisions. Se priver d'une appréciation chiffrée des proportions globales ne serait certainement pas rendre service à la théorie, ni à l'entreprise générative tout court.

\section{Conclusion}

L'objet de cette introduction était d'identifier les propriétés naturelles de la phonologie qui font qu'elle se démarque des autres disciplines linguistiques. Saussure (1915: 23ssq) explique que le métier de linguiste est plus difficile que celui de physicien, chimiste ou biologiste car le linguiste, avant de pouvoir exercer son activité, doit définir l'objet de son étude. La Langue, dit Saussure, est voilée par un bruit de fond provenant de la Parole, et il s'agit de l'en libérer car non seulement elle est le véritable objet de la linguistique, mais encore elle doit en être le premier: il est vain de vouloir l'atteindre à travers l'étude de la Parole ( $c f$. note 1). Milner (1989 : 38ssq) met en perspective cette obligation du linguiste de construire son objet avant de pouvoir agir.

Mon intention ici était de montrer que la phonologie est à la linguistique ce que celle-ci est aux autres sciences : le vilain petit canard qui marche sur des œufs parce qu'il risque constamment d'étudier des faits qui ne relèvent pas de ses attributions. Là où les autres linguistes peuvent commencer leur travail, le phonologue doit d'abord épurer les observables des facteurs phonétiques, lexicaux, diachroniques et analogiques.

Il ne peut pas se prévaloir des arguments dont les syntacticiens se servent pour lutter contre ceux qui professent que le langage humain est fait de Parole et de Parole seulement (p. ex. les approches « exemplaristes » ou « usage based» de type Bybee 2001), ainsi que contre ceux qui nient qu'une forme 
de Grammaire Universelle existe (tout court, ou seulement en phonologie comme p. ex. Carr 2000) ${ }^{35}$.

La différence naturelle de la phonologie procède donc de quatre propriétés: 1) sa situation à l'interface avec la phonétique, 2) son enchevêtrement avec le lexique (et, partant, avec la diachronie et l'analogie), 3) l'absence de récursivité en son sein (d'où le nombre seulement fini des unités étudiées), 4) la présence d'un système (et donc de pression systémique) qui n'existe pas ailleurs. C'est en cela, je pense, que la phonologie est différente, et non pour les raisons exposées dans Bromberger \& Halle (1989).

Il est vrai que certains aspects de cette introduction dépassent le cadre d'une mise en bouche pour le volume de la revue Corpus consacré à l'usage et l'utilité des corpus en phonologie. Cependant, les particularités de la phonologie dont il a été question ont des conséquences directes sur le statut des corpus dans cette discipline. La plus importante procède de l'absence de récursivité et du fait que l'objet d'étude est un ensemble fini d'unités : le corpus, dans ces conditions, n'est pas un luxe en phonologie; il est une obligation.

Ensuite, la présence de forces systémiques en phonologie fait qu'on peut, qu'on doit faire la différence entre des corpus structuralistes et des corpus générativistes. J'ai dit le danger que revêt la pratique fort populaire ces dernières années qui consiste à compiler des données de langues qu'on ne connaît pas et qui sont puisées dans des grammaires qu'on n'a pas lues entièrement et que l'on ne sait évaluer. Je pense que l'ambition générative à découvrir les universaux doit s'accompagner d'un contrôle structuraliste. Toutes les contributions à ce volume sont d'ailleurs basées sur des corpus structuralistes.

Enfin, le bruit de fond phonétique, lexical, diachronique et analogique produit autant d'obstacles à l'utilisation du corpus

35. Les forces behaviouristes et anti-saussuriennes, un siècle presque après la publication du Cours, n'ont pas disparues, loin de là. Elles se réincarnent perpétuellement. C'est leur relative visibilité et leur acceptation dans l'agora linguistique qui est variable, plutôt que leur existence. Katz \& Bever (1974) ont écrit un article fort intéressant sur la vie cyclique des forces anti-saussuriennes. 
Présentation. En quoi la phonologie est vraiment différente

en phonologie: il faut d'abord le nettoyer avant de pouvoir l'utiliser. Or ce qui est précisément considéré comme un bruit de fond qu'il s'agit d'éliminer est sujet à débat et dépend largement des orientations théoriques épousées par l'analyste. Sauf peutêtre l'influence analogique pour laquelle tous s'accorderont qu'elle n'a pas sa place dans l'analyse phonologique (encore que Joaquim Brandão de Carvalho ne soit peut-être pas d'accord ici), il y a des avis fort divergents sur la question de savoir quelle partie du gâteau exactement relève de facteurs phonétiques, lexicaux et diachroniques. Il en découle une exigence théorique plus importante que la phonologie impose au phonologue: celui-ci doit avoir une position théorique explicite avant de commencer le travail sur les matériaux bruts. En d'autres mots, les corpus phonologiques sont plus orientés, plus théoriques et plus construits que ceux qui servent dans d'autres disciplines. Ils sont pour cette raison plus difficilement réutilisables.

\section{Présentation des contributions}

Cette longue introduction permet de dégager une grille d'analyse pour le classement des contributions à ce volume. Examinons d'abord ce que toutes ont en commun.

En premier lieu une banalité, mais qui mérite mention : le corpus est un outil qui sert le propos d'une analyse phonologique - il n'est pas une fin en soi.

L'ancrage du côté structuraliste ensuite : tous les corpus présentés ici enquêtent sur un aspect particulier d'une seule langue, et sont donc structuralistes. Aucun corpus n'est générativiste dans ce sens qu'il essaierait de fournir des données typologiques cross-linguistic. Cela vaut également, je pense, pour la contribution de Jacques Durand et Julien Eychenne, qui compare le comportement de trois systèmes (Languedoc, Pays Basque, Vendée) au regard d'un aspect particulier du français, l'opposition entre des mots avec et sans schwa final (le couple mer et mère constitue-t-il une paire minimale?). La classification du corpus en question comme corpus générativiste serait indue, me semble-t-il, car celui-ci allie des aspects typologiques au contrôle structuraliste. C'est là la force du 
projet PFC, qui a été conçu précisément dans cette perspective (Durand et al. 2002, Durand \& Lyche 2003) : un maillage de points d'enquête couvre des variétés d'une même «langue " tout en portant une attention particulière aux propriétés systémiques de chaque variété enregistrée. Un autre point essentiel qui permet seulement la comparaison entre les différents points du réseau est le cadre méthodologique commun de toutes les enquêtes : le protocole est strictement identique partout.

La question de savoir si les systèmes particuliers enregistrés sont des variétés (des dialectes) d'une même langue - le français -, ou si au contraire ils ont le statut de langues différentes n'en est pas vraiment une car chacun sait que «la langue» n'est pas une catégorie linguistique pertinente. Il suffira de dire que les systèmes mis en concurrence entretiennent un rapport génétique et diachronique assez étroit pour que leur comparaison soit raisonnable et produise des résultats utilisables. Sans ambition dialectologique, qui supposerait un maillage beaucoup plus fin et une méthodologie quelque peu différente, le PFC en général et la contribution de J. Durand et J. Eychenne en particulier se présentent donc comme un exemple de ce que j'ai appelé de mes vœux plus haut: procédant du milieu génératif, une visée typologique (cross-linguistic) est mise en œuvre sous contrôle systémique.

Il va de soi que ce qui vient d'être dit a fortior $i$ vaut pour la contribution de M.-J. Dalbera-Stefanaggi et J.-Ph. Dalbera, qui travaillent sur des corpus proprement dialectologiques et donc structuralistes par définition - surtout dans le cas de la BDLC et du THESOC, corpus dont les auteurs soulignent « l'épaisseur».

Enfin, toutes les contributions à ce volume sont basées sur des corpus lexicographiques, non textuels - à l'exception, toujours, de J. Durand et J. Eychenne d'une part, et M.-J. Dalbera-Stefanaggi et J.-Ph. Dalbera d'autre part. Pour les premiers, ceci tient à la nature même de l'objet d'étude, qui se trouve être un phénomène de sandhi. On voit mal comment on pourrait étudier ce qui se passe au contact entre deux mots sans les mettre en contexte. Par ailleurs, le phénomène étudié suppose un contrôle sociologique des locuteurs et de leur production qu'il est difficile de satisfaire hors contexte. 
On le voit, la proportion des corpus lexicographiques et textuels au sein du présent volume (six contre deux, en comptant la BDLC et le THESOC du côté textuel) est représentative de la nature de la phonologie qui a été dégagée plus haut : contrairement à d'autres disciplines, la phonologie procède du lexique et entretient avec lui des relations étroites et compliquées. Aussi a-t-elle une prédilection naturelle pour les corpus lexicographiques.

Le second critère à la lumière duquel les dix contributions peuvent être lues est la spécificité qui procède de l'ancrage lexical de la phonologie : les observables doivent être nettoyés des facteurs lexicaux, diachroniques et analogiques avant de pouvoir informer l'analyse. Nul n'y échappe, et les dix articles mènent une lutte plus ou moins acharnée avec la vicissitude de leur objet, essayant de définir ses relations avec le lexique, la diachronie et l'analogie.

L'article de Joaquim Brandão de Carvalho est entièrement consacré à l'analogie : il existe en portugais une classe de mots qui au singulier est homogène, mais produit des pluriels différents où la voyelle radicale $-o$ - soit demeure identique à celle du singulier, soit devient -ATR. L'appartenance d'un mot à la classe alternante ou nonalternante, à l'origine, est visiblement fonction du timbre de sa voyelle en latin. Le conditionnement d'origine correspondait à une logique phonétique et a laissé, après sa mort, des ruines lexicales qui ont alors commencé à danser dans le vent analogique. Celui-ci a soufflé dans les deux sens, rendant alternants des mots qui n'ont pas de raison latine d'alterner, et faisant adhérer à la classe non-alternante des mots dont l'origine interdit l'appartenance. Il est évident que seul un bilan chiffré portant sur l'ensemble du lexique de cette classe particulière (238 formes en tout) permet de savoir, et encore à grand mal, quelle est la proportion, dans les deux classes - alternante et non-alternante - des unités qui représentent une formation analogique. La question qui alerte l'auteur, ici, est la tendance de l'évolution, qui va à l'encontre du rapport de force numérique: la classe alternante, bien que minoritaire en nombre, non seulement survit sans se faire assimiler par des 
forces lexicales supérieures, mais encore gagne du terrain en arrachant des unités à celles-ci. Il existe donc en portugais, selon J. Brandão de Carvalho, deux régularités bien vivantes et concurrentes : celle qui produit des pluriels alternants, et celle dont le résultat est non-alternant. C'est le statut de ces « régularités » qui est important: il ne s'agit pas de règles au sens de SPE (ou dans celui des lois phonétiques néogrammairiennes), car le conditionnement contextuel est mort depuis longtemps. On parle bien ici d'analogie - mais " d'analogie régulière et vivante ", qualificatif qui semble être une contradiction dans les termes. C'est cette notion d'analogie lexicalement désincarnée que l'auteur développe et, contre l'acception traditionnelle (qui est aussi celle que j'ai utilisée en section 3), entend installer dans l'architecture générale de la grammaire. Il existerait ainsi un type d'analogie qui transforme $\mathrm{X}$ en $\mathrm{Y}$ sans prendre appui sur aucune unité lexicale particulière.

L'article d'Edmund Gussmann n'est pas à proprement parler une étude de corpus au sens classique, qui suppose une liste de mots ou d'énoncés. C'est, en revanche, une étude concernant un corpus de données, certes disparates et appartenant à plusieurs paradigmes, voire plusieurs langues différentes, mais ayant ceci d'uniforme qu'elles éclairent un phénomène unitaire - ou qui est pris pour tel plutôt - du polonais : la palatalisation des consonnes vélaires.

L'objectif de l'auteur est de montrer que le phonétisme dont il a été question plus haut (sections 1 et 2.3 à 2.7 ) est sans fondement. Un processus peut bien être conditionné par un contexte phonétiquement plausible - comme l'exemple choisi ici, la palatalisation de $[\mathrm{k}, \mathrm{g}, \mathrm{x}]$ devant voyelle antérieure ; pour autant il n'est pas nécessairement décidé dans la bouche par des mécanismes de coarticulation, d'aérodynamique ou autres. Il peut tout aussi bien avoir son origine dans le cerveau, car les propriétés phonétiques d'un son ne sont que le reflet de sa substance phonologique qui, elle, est définie en termes de primitives phonologiques, sises dans le cerveau. Il s'agit donc d'un chapitre du débat entre phonétique et phonologie : existe-til une part du gâteau qui est décidée par la seule phonologie (la 
Langue saussurienne), et si elle existe, quelle est sa taille? E. Gussmann se place résolument du côté « abstrait », c'est-à dire de ceux qui, à l'instar de Stephen Anderson (1981) («Why phonology isn't natural») et Jonathan Kaye (1989: 42-49) ( The nonphonetic bases of phonological phenomena»), pensent que les phénomènes phonologiques existent pour des raisons qui n'ont aucun rapport avec la phonétique ou la bouche.

C'est donc la palatalisation des consonnes vélaires que l'auteur a retenue pour montrer le caractère inopérationnel du principe phonétique. Ce phénomène a été choisi à dessein car sa motivation phonétique, au demeurant, tombe sous le sens. E. Gussmann montre alors, au fil d'un minutieux examen de tous les recoins de la phonologie du polonais et de ses dialectes, que l'hypothèse phonétique ne mène à rien: elle surgénère et sousgénère à la fois. Certaines palatalisations en effet ne surviennent pas là où elles devraient se produire, d'autres, en revanche, surgissent sans motivation phonétique. L'auteur conclut qu'elles ne sont pas décidées par un mécanisme aveugle, mais de manière réfléchie et sélective - donc dans le cerveau. L'outil qui permet à l'auteur d'affirmer cela est un examen complet des données qui, lors d'analyses précédentes du phénomène, a fait défaut et ainsi promu la vision phonétique. C'est ce florilège de données, disparates au demeurant, qui peut être considéré comme un corpus - ou un corps de données.

Mon propre article propose de reconnaitre (au moins) deux fonctions distinctes du corpus : heuristique et validante. Chacun connaît la seconde: un corpus est utilisé afin de démontrer la plausibilité ou la véracité d'une hypothèse qui a une existence indépendante. A la différence de celle-ci, la première montre, plutôt que de démontrer. Le linguiste ne cherche pas ici à convaincre, mais à comprendre. Il part à la rencontre de l'inconnu avec un questionnement, mais sans solution, même hypothétique, pour le puzzle dont il a identifié quelques pièces. C'est le corpus lui-même qui suggérera des hypothèses et, idéalement, la solution. Le corpus agit ainsi en tant que conseiller qui propose, jette une nouvelle lumière sur la « réalité » et restreint les analyses possibles. 
La vertu du corpus qui lui permet d'exercer cette fonction est consubstantielle à son existence: le classement. Qui dit corpus, en effet, dit principe de classement. Or c'est un classement selon le ou les critères pertinents qui montrera la " réalité » sous le jour qui produira le déclic chez le linguiste. Aussi conclut-on qu'il n'existe pas, en linguistique ou ailleurs, de corpus sans projet d'exploitation ou activité théorique préalable : le corpus, heuristique autant que validant, est un objet orienté, donc théorique.

Le corpus heuristique a ceci de particulier qu'il est éphémère : sitôt ses services rendus, il sera transformé par le linguiste en corpus de validation (qui aura vocation à convaincre le monde). Ce changement d'objectif induit alors une organisation et une présentation très différentes de celles qui ont permis la découverte du fonctionnement linguistique en question. L'article entend donc, aussi, sortir le corpus heuristique de l'ombre des cabinets et des disques durs, et le garantir contre l'oubli, oubli souvent parfait auprès de ceux-là même qui l'ont créé. Ce faisant, j'essaie d'isoler les propriétés invariables des corpus heuristiques: le moment de leur intervention dans le processus de découverte (lorsque la recherche est au point mort), les situations dans lesquelles ils sont utiles et celles où ils ne sont d'aucun secours.

Ce propos est illustré par un cas particulier tiré de mon expérience personnelle, i.e. les alternances de longueur vocalique dans les itératifs tchèques. Les principales difficultés ici sont de deux ordres : le fait que le corpus à établir est certes lexicographique mais doit contenir des paires de mots - itératif et non-itératif-, et le statut des paires « diachroniques », i.e. qui n'entretiennent pas de relation itérative en synchronie, mais où tout porte à croire que tel était le cas dans le passé.

Xavier Barillot enquête sur les verbes du somali qui présentent trois consonnes. Il montre, grâce au comportement des différents verbes au regard d'une alternance voyelle - zéro, qu'en réalité il existe de vrais et de faux trilitères : ceux dont la forme sous-jacente possède réellement trois consonnes, et ceux qui en comportent quatre. Cette analyse fait également une prédiction concernant l'identité des deux voyelles des vrais 
trilitères. Ce diagnostic en point de mire, l'auteur construit un corpus électronique qui compile plusieurs dictionnaires du somali, ainsi que des outils d'interrogation. Ayant ainsi une vue assez complète du lexique, il peut produire un bilan chiffré des paradigmes en question. Celui-ci montre qu'il existe des contreexemples en quantité non-négligeable, mais qui se réduisent à la portion congrue si on prend en compte des processus segmentaux indépendants.

Enfin, X. Barillot se met sur la trace d'un nombre important de faux trilitères, ceux qui possèdent, outre les deux consonnes périphériques, une géminée médiane. Tous partagent la même vocalisation $a-i$, alors que $a-a$ est attendu. Il s'agit ici visiblement d'emprunts à l'arabe égyptien voisin, et plus particulièrement à la forme II de l'arabe qui possède, justement, une géminée médiane. L'auteur compare les formes somalies et arabes une à une, constatant une parfaite concordance en ce qui concerne la géminée et son adaptation en somali, mais une incohérence déconcertante du vocalisme. La solution qu'il propose alors est la suivante : le $i$ de la mélodie somalie $a-i$ n'a pas été emprunté mais résulte d'une activité épenthétique propre au somali (le $i$ y fait plus généralement office de voyelle épenthétique).

Les deux études - celle qui valide et chiffre la suspicion initiale et celle qui porte sur les emprunts - supposent l'appui d'un corpus. X. Barillot explique à la fin de sa contribution quels sont les problèmes qu'il a rencontrés lors de la compilation de plusieurs dictionnaires, ainsi que le codage qui en a résulté pour la base de données finale.

L'article de Gilles Boyé et Patricia Cabredo Hofherr fait découvrir au public une corrélation entre la voyelle radicale et la voyelle thématique des verbes en espagnol qui jusqu'alors n'avait pas été identifiée dans cette langue pourtant bien étudiée. En développant systématiquement une suspicion de James Harris à propos d'une asymétrie distributionnelle limitée, les auteurs montrent qu'il existe un lien de nécessité entre la voyelle radicale (appelée pré-thématique par précaution) et la voyelle thématique. Ceci à condition de bien vouloir mettre la voyelle radicale à nu : elle est sujette à plusieurs alternances 
croisées, et ce n'est qu'en identifiant sa forme sous-jacente (ou forme par défaut, terme préféré par les auteurs) que l'on peut entrevoir la distribution complémentaire. Celle-ci étant acquise, se pose le problème de la poule et de l'œuf : est-ce, à l'image de la métaphonie romane bien connue, la voyelle thématique qui influence la voyelle radicale, ou est-ce que celle-ci au contraire met la voyelle thématique au pas? Les auteurs optent pour la dernière solution et motivent leur choix.

Le corpus que les auteurs construisent montre la validité de ce fonctionnement pour l'ensemble du lexique de l'espagnol: il s'agit de rassembler tous les verbes et de les classer en fonction de leurs propriétés lexicales. Pour ce faire, il est nécessaire de mettre en place un système classificatoire inédit qui croise de multiples critères. Se pose enfin la question de savoir si le conditionnement intervocalique mis à jour est synchroniquement actif, ou si on a affaire aux vestiges d'un système fossilisé.

L'article d'Olivier Rizzolo décrit le šatrovački. Il s'agit d'un langage secret/ludique basé sur le serbo-croate qui, comme le verlan en France, intervertit les «syllabes». L'objectif ici est de mettre à la disposition de tous des données qui n'ont jamais été rendues accessibles, et d'en étudier un aspect particulier. L'auteur a rassemblé un corpus de 194 mots šatrovački et explique les écueils rencontrés lors de cette entreprise. Il faut d'abord trouver des locuteurs, ce qui suppose que l'on sache définir cette notion pour un langage secondaire. Car le travail avec des informateurs suppose que ceux-ci, au même titre que les locuteurs d'une langue naturelle, soient productifs et sachent transformer tout mot serbo-croate en šatrovački.

Le corpus présenté est orienté: en dehors des présupposés normaux, il l'est à l'image du PFC où un petit nombre de phénomènes sur lesquels portera l'attention a été défini avant même que le recueil des données ne commence. O. Rizzolo a donc constitué son corpus en vue d'étudier trois événements : 1) une épenthèse de schwa (hleb $\rightarrow$ bəhle), 2) la modification d'une voyelle (kafa $>$ fuka) et 3) l'existence de marques flexionnelles à l'intérieur des mots verlanisés (pričam 
« je parle » où $-m$ marque la $1^{\text {ère }}$ personne du singulier devient čampri, et non čaprim).

Le phénomène dont l'auteur propose ici une analyse est l'apparition inopinée de schwa. Celle-ci est remarquable en tant que telle parce que ce son (ou ce phonème) est absent du serbocroate : comment se fait-il que les locuteurs choisissent une voyelle qu'ils ne connaissent pas lorsque la phonotactique d'un langage secondaire en appelle une et que n'importe quelle voyelle ferait l'affaire?

L'auteur pose ensuite la question de savoir à quelle logique l'épenthèse répond. On relève une constante dans tous les cas: l'épenthèse se trouve toujours au même endroit, i.e. après la dernière consonne du mot dans sa forme d'origine. Ce sont les unités $\mathrm{C}_{1} \mathrm{VC}_{2}$ qui provoquent l'insertion: la verlanisation produit $\mathrm{C}_{2} \partial \mathrm{C}_{1} \mathrm{~V}$. Or si le groupe initial $\|_{2} \mathrm{C}_{2}$ était la cause de l'épenthèse, l'insertion d'une voyelle avant $\mathrm{C}_{2}$ serait une solution tout aussi satisfaisante. On ne l'observe jamais. O. Rizzolo y voit un indice fort pour l'existence d'une position vocalique vide après les consonnes finales des mots nonverlanisés. Il s'agit là en réalité d'une position qui est postulée par la Phonologie de Gouvernement.

Enfin, l'auteur se pose la question de savoir si l'épenthèse est due à la complication consonantique en début de mot qui est créée par la verlanisation $\left(\mathrm{C}_{1} \mathrm{VC}_{2}>\mathrm{C}_{2} \mathrm{C}_{1} \mathrm{~V}\right)$, ou si le mécanisme de verlanisation n'accepte que des unités bisyllabiques et contraint donc tout objet CVC à se doter d'une voyelle supplémentaire avant la transformation. La solution retenue est la dernière, notamment parce qu'un certain nombre de groupes $\#_{2} C_{1}$ initiaux existent par ailleurs dans la langue sans que celle-ci ne réagisse.

Jacques Durand et Julien Eychenne, comme il a été indiqué, enquêtent à propos du caractère distinctif de schwa en position finale de mot : est-ce que, à l'écoute, mer et mère sont ou non confondus? Après une présentation du problème et de son rôle dans l'étude du français, les auteurs introduisent les traits généraux du projet PFC dans lequel leur contribution se situe. La construction et le codage du corpus concernant le schwa final sont ensuite expliqués. Enfin, le résultat de trois 
points d'enquête est présenté : une variété languedocienne (Aude) est comparée à un idiome du Pays Basque (Biarritz) et à un corpus vendéen. Les auteurs exposent également les paramètres sociaux pertinents. Le résultat est graduel : si en Languedoc le schwa final est bien distinctif, il ne l'est plus du tout en Vendée malgré sa possible mobilisation phonétique. Le système de Biarritz représente une situation intermédiaire en pleine évolution où différents locuteurs par exemple peuvent montrer des comportements variés.

Enfin, une régularité de type fonctionnel se dessine au fil des analyses. Les auteurs ont également vérifié les éventuelles épenthèses de schwa en sandhi lorsque la frontière de mot est entourée de plusieurs consonnes (type Marc [ə] Blanc). Or il semble que l'épenthèse est corrélée au caractère distinctif de schwa final: elle est absente dans la variété de l'Aude (qui est pourtant réputée avoir " beaucoup » de schwas) où le schwa final demeure bien en place, alors qu'elle se manifeste en Vendée (idiome "nordique » où le schwa est pourtant censé se raréfier) où le schwa final a disparu. Il sera intéressant de vérifier ailleurs si l'épenthèse est vraiment bloquée tant que le schwa final n'a pas perdu sa valeur distinctive.

L'article de Claudine Pagliano retrace avec précision la construction d'un corpus qui a l'ambition de réunir tous les mots $\mathrm{du}$ français présentant une consonne épenthétique - i.e. nonétymologique - à l'intersection entre le radical et un suffixe (p.ex. numéro / numéro-t-er). Les écueils, ici aussi, relèvent des trois facteurs diachronique, lexical et analogique. Puisque l'étude veut isoler les consonnes issues d'une activité épenthétique, il s'agit d'abord de déterminer si les consonnes candidates ont une base étymologique ou non. Il faut ensuite savoir quel est le statut dérivationnel des mots qui portent une consonne potentiellement épenthétique: ils seront comptabilisés s'ils représentent une entrée lexicale autonome, mais doivent être négligés au cas où ils ne sont que dérivés de mots ayant déjà subi au préalable l'épenthèse. Enfin, les consonnes intruses peuvent aussi procéder d'une activité analogique, option qu'il convient d'examiner cas par cas. 
S'ajoute à ces problèmes le caractère productif de l'épenthèse : celle-ci crée des mots éphémères qui soit vivent le temps de leur prononciation, soit n'ont pas (encore) droit de cité dans les dictionnaires (p.ex. stabilo-t-er, tennis-t-ique). Le corpus de C. Pagliano se nourrit donc de sources variées, dictionnairiques, journalistiques, orales ou relevant de langages spécialisés. Le résultat principal en est la désillusion pour ceux qui croient, selon toute bonne intuition et expérience, que les consonnes épenthétiques existent afin de casser l'hiatus : s'il est vrai qu'elles sont toujours suivies d'une voyelle, on en trouve aussi après consonne, et il s'agit là de $10 \%$ des épenthèses (p.ex. atlastique, noirdure, briqueter). Seul un corpus qui permet de faire des bilans chiffrés peut invalider l'hypothèse naturelle de l'hiatus. C. Pagliano s'oriente alors vers une autre solution, morphologique. Elle montre que les épenthèses ont un rapport avec le suffixe: certains génèrent des consonnes intruses, d'autres non. A l'instar d'autres langues où différentes classes de suffixes ont des effets variés (level 1 vs. level 2 suffixes dans la terminologie de la Phonologie Lexicale), la consonne épenthétique serait donc la marque tangible d'une classe morphologique. Partant de cette idée, l'auteur développe une analyse qui concilie la cause morphologique avec son effet phonologique : c'est in fine l'interface des deux modules qui est visé par cette étude.

Jean-Philippe Dalbera et Marie-José DalberaStefanaggi parlent de phonologie indiscrète, notion qui paraît être une contradiction dans les termes. Elle exprime la conclusion à laquelle les auteurs arrivent en analysant le continuum qui se présente au dialectologue naviguant sur les vagues de grands corpus tels la BDLC (Banque de donnée langue corse) et le THESOC (Thésaurus Occitan). Rien n'est discret ici, l'un donne dans l'autre sans cesse : la synchronie est en même temps de la diachronie et vice-versa; la compétence d'un locuteur se révèle être multiple car il peut osciller entre plusieurs dialectes; les dialectes eux-mêmes ne sont pas « isoglossables », leur transition est permanente et non discrète ; même le phonème, unité discrète par excellence, peut prendre une forme telle qu'on est tenté de lui refuser cet attribut : c'est le 
cas lorsque ses réalisations phonétiques se confondent avec celles d'autres phonèmes, situation que les auteurs illustrent par un cas concret. Le résultat est un défi lancé à la phonologie, ou plutôt à la linguistique toute entière : le discret existe-t-il ? Tout en affirmant que la discontinuité est vraisemblablement une construction interprétative des locuteurs et les linguistes, les auteurs ne vont pas jusqu'à sceller la mort du phonème, qu'ils entendent « relativiser sans mettre en doute son existence ».

C'est donc la question du réalisme cognitif des objets théoriques qui est posée. Le structuralisme, que les auteurs épousent, a connu les deux positions : celle qui, au nom d'une description exacte du système, ne se soucie pas de la localisation des objets postulés (dans le cerveau ou sur la planète mars) (Hjelmslev 1948 par ex.). Face à celle qui croit en la réalité "psychologique » des phonèmes (p. ex. Sapir 1993, Hockett 1948). En déclarant le phonème une vue d'esprit à la fois du locuteur et du linguiste, les auteurs en réalité peuvent se réclamer des deux traditions : le produit de la construction du locuteur est nécessairement sis dans son cerveau - le phonème a donc une réalité cognitive. De l'autre côté, le linguiste le construit aussi, mais les deux constructions ne se superposent pas: celle du linguiste ne doit pas se mesurer à l'aune du système cognitif.

Le lecteur assiste ici à la mise en musique d'une différence bien connue entre les conceptions saussurienne et chomskienne de la Langue / compétence: «la Langue n'est complète dans aucun [cerveau], elle n'existe parfaitement que dans la masse » dit Saussure (1915: 30), alors que le seul théâtre de la compétence chomskienne est le cerveau. Qu'est donc l'objet de la linguistique ? Doit-elle chercher à caractériser un objet naturel qui a un support biologique, le cerveau, et dont la partie centrale, la Langue / compétence, se laisse réduire à des invariants partagés par tous les locuteurs et qui sont donc biologiquement matériels ? Ou la Langue / compétence est-elle un objet qui ne peut se caractériser pleinement qu'en considérant l'ensemble des cerveaux des locuteurs? Il me semble qu'il est contradictoire de faire de la Langue un objet socialement divers car elle est, précisément, ce qui ne varie point d'individu en individu. Dans ces conditions, elle ne peut 
être biologiquement désincarnée, et la caractérisation à laquelle aboutit le linguiste n'a de sens que si elle rejoint celle du locuteur. Je pense que le mot « social », chez Saussure, renvoie non pas à de la variation, mais au fait qu'un seul individu ne peut modifier la Langue (alors qu'il est maître de sa Parole) : il faut pour cela l'assentiment (tacite et inconscient bien sûr) de la communauté entière. Saussure (1915: 31) précise en effet que la Langue «est la partie sociale du langage, extérieure à l'individu, qui à lui seul ne peut ni la créer ni la modifier».

Si le réalisme cognitif (ou social) des objets théoriques est la question centrale posée par M.-J. Dalbera-Stefanaggi et J.-Ph. Dalbera, leur article ne s'y résume pas pour autant. Les auteurs passent en revue les différentes fonctions des grands corpus et montrent où, comment, pour quelles raisons et dans quelles circonstances ils rendent des services dont le linguiste aurait dû se passer à leur défaut. Ils montrent, à travers une série d'anecdotes et de faits dont la lénition et la fortition corses sont les plus saillants, que l'établissement des faits, la validation d'hypothèses et leur altération (fonction heuristique) ne se déroulent pas de la même manière selon qu'on met ou non à contribution les grands corpus. La fin n'étant jamais bien loin des moyens, les auteurs entendent montrer, in fine, que la conclusion à «l'indiscrétion» est notamment l'œuvre des grands corpus : c'est eux qui mettent en difficulté le discret.

Enfin, l'article de Jean-Léo Léonard présente le cycle de vie d'un corpus dialectal qui recense le système vocalique d'une variété d'oïl, celle de l'île de Noirmoutier et de ses environs. L'objectif constant de l'auteur, depuis la première description en 1991 jusqu'à la plus récente, est de dégager, à partir de transcriptions à l'oreille et d'enregistrements, le système vocalique sous-jacent noirmoutrin qui se caractérise notamment par une série de diphtongues nourrie.

Trois étapes sont décrites: d'abord la constitution du corpus en 1991. Celle-ci a donné lieu à la construction d'une première hypothèse suivant la méthode classique en dialectologie qui s'oriente du côté de la diachronie : les formes modernes sont le résultat des formes anciennes qui ont été modifiées. Le même corpus est ensuite apprécié, en 1999, à la 
lumière d'une théorie monovalente de la structure interne des voyelles. Celle-ci apporte quelques modifications à l'hypothèse initiale. Enfin, l'épisode le plus récent revient aux données brutes: l'auteur contrôle le résultat en soumettant les enregistrements originaux à l'analyse instrumentale qui a fait des progrès depuis 1991 et désormais met à la portée de tous la visualisation de menus détails. L'étude à l'aide du logiciel Praat est intéressante en ceci qu'elle confirme assez largement les résultats du système sous-jacent que l'auteur a construit en 1991 sur la foi des transcriptions à l'oreille.

\section{Références bibliographiques}

Les mentions suivantes à la fin d'une référence indiquent que celle-ci est disponible en ligne :

WEB : www.unice.fr/dsl/tobias.htm

ROA : http://roa.rutgers.edu

Anderson S. (1981). « Why phonology isn't natural », Linguistic Inquiry $12: 493-539$.

Anderson S. (1985). Phonology in the Twentieth Century. Chicago : University of Chicago Press.

Anderson S. (2000). «Reflections on 'On the Phonetic Rules of Russian' », Folia Linguistica 34 : 11-28.

Angoujard J.-P. (1997). «La Phonologie Déclarative», Langages $125: 35-54$.

Archangeli D. (1988). "Aspects of underspecification theory », Phonology 5 : 183-208.

Bailey T. (1995). Nonmetrical Constraints on Stress. Thèse de doctorat, Université du Minnesota. Base de données disponible à http://www.cf.ac.uk/psych/ssd/index.html.

Beckman J. (1997). « Positional Faithfulness, Positional Neutralisation and Shona Vowel Harmony ", Phonology 14 : 1-46.

Blevins J. (2004). Evolutionary Phonology. Cambridge: Cambridge University Press.

Bloomfield L. (1939). "Menomini morphophonemics", Travaux du Cercle Linguistique de Prague 8 : 105-115. 
Présentation. En quoi la phonologie est vraiment différente

Borowsky T. (2000). «Word-faithfulness and the direction of assimilation », The Linguistic Review 17 : 1-28.

Bourdieu P. (1972). Esquisse d'une théorie de la pratique, précédé de Trois études d'ethnologie kabyle. Genève: Droz.

Bromberger S. \& Halle M. (1989). «Why Phonology Is Different », Linguistic Inquiry 20 : 51-70.

Bruck A., Fox R. \& La Galy M. (éds.) (1974). Papers from the parasession on natural phonology. Chicago: Chicago Linguistic Society.

Bybee J. (2001). Phonology and Language Use. Cambridge: Cambridge University Press.

Campbell L. (1981). «Generative phonology vs. Finnish phonology : retrospect and prospect », in Didier Goyvaerts (éd.) Phonology in the 80's. Ghent: Story-Scientia, pp. $147-182$.

Carr, P. (2000). « Scientific Realism, Sociophonetic Variation, and Innate Endowments in Phonology », in N. BurtonRoberts, P. Carr \& G. Docherty (éds.) Phonological Knowledge. Conceptual and Empirical Issues. Oxford: Oxford University Press, pp. 67-104.

Carr P. (2003). «Innateness, internalism and input: Chomskyan rationalism and its problems ", Language Sciences $25: 615-635$.

Casali R. (1996). Resolving Hiatus. Thèse de doctorat, UCLA.

Charette M. \& Göksel A. (1994). «Vowel Harmony and Switching in Turkic languages », SOAS Working Papers in Linguistics and Phonetics. $4: 31-52$. Egalement in Kardela Henryk, Bogdan Szymanek (éds.) (1996), A Festschrift for Edmund Gussmann. Lublin: University Press of the Catholic University of Lublin, pp. 29-56.

Charette M. \& Göksel A. (1996). «Licensing constraints and vowel harmony in Turkic languages ", SOAS Working Papers in Linguistics and Phonetics $6: 1-25$. Egalement in Cyran Eugeniusz (éd.) (1998), Structure and Interpretation. Studies in Phonology. Lublin : Folium, pp. 65-88. 
Chomsky N. (1951). The Morphophonemics of Modern Hebrew. Masters thesis, Université de Pennsylvania.

Chomsky N. (1957). Syntactic Structures. Mouton : La Haye.

Chomsky N. (1959). « Review of Skinner's Verbal Behavior », Language 35 : 26-58.

Chomsky N. (1965). Aspects of the Theory of Syntax. Cambridge, Mass. : MIT Press.

Chomsky N. (1973). "Conditions on Transformations », in S. Anderson \& P. Kiparsky (éds.) A Festschrift for Morris Halle. New York: Holt, Rinehart \& Winston, pp. 232-286.

Chomsky N. (1995). The Minimalist Program. Cambridge, Mass. : MIT Press.

Chomsky N. (2001). « Derivation by Phase », in M. Kenstowicz (éd.) Ken Hale: A Life in Language. Cambridge, Mass. : MIT Press, pp. 1-52.

Chomsky N., Hauser M. \& Fitch T. (2002). «The faculty of language : what is it, who has it, and how did it evolve? », Science 298 : 1569-1579.

Clements G. (1988). " Toward a Substantive Theory of Feature Specification ", in J. Blevins \& J. Carter (éds.) Proceedings of NELS 18. Amherst: GLSA, Vol 1, pp. 79-93.

Clements G. (2001). « Representational economy in constraintbased phonology », in T. Hall (éd.) Distinctive Feature Theory. Berlin, New York : Mouton de Gruyter, pp. 71-146.

Clements G. (2002). Economie de traits et représentations lexicale. Communication présentée aux 4è Journées Internationales du GDR Phonologies, Grenoble juin 2002.

Cole J. (1995). "The Cylcle in Phonology», in J. Goldsmith (éd.) The Handbook of Phonological Theory. Oxford: Blackwell, pp. 70-113.

Coleman J. (1995). "Declarative lexical phonology», in J. Durand \& F. Katamba (éds.) Frontiers in Phonology. Atoms, Structures, Derivations. London \& New York: Longman, pp. 333-382. 
Présentation. En quoi la phonologie est vraiment différente

Coleman J. (1998). Phonological Representations: Their Names, Forms and Powers. Cambridge: Cambridge University Press.

Coleman J. (2002). «Phonetic Representations in the Mental Lexicon ", in B. Laks \& J. Durand (éds.) Phonetics, Phonology and Cognition. Oxford: Oxford University Press, pp. 96-130.

Dalbera J.-P. (2002). «Le corpus entre données, analyse et théorie », Corpus $1: 89-104$.

de Lacy P. (2002). The formal expression of markedness. Thèse de doctorat, Université du Massachusetts.

Dell F. (1973). Les règles et les sons. Paris : Hermann.

Dinnsen D. (1980). «Phonological rules and phonetic explanation », Journal of Linguistics 16 : 171-191.

Donegan P. (1978). The natural phonology of vowels. Thèse de doctorat, Ohio State University. Publiée par Garland Press, New York 1985.

Donegan P. \& Stampe D. (1978). «The Syllable in Phonological and Prosodic Structure », in A. Bell \& J. Hooper (éds.) Syllables and Segments. Amsterdam: NorthHolland, pp. 25-43.

Donegan P. \& Stampe D. (1979). «The study of natural phonology », in D. Dinnsen (éd.) Current approaches to phonological theory. Bloomington: Indiana University Press, pp. 126-173.

Dresher E. (1981). «Abstractness and Explanation in Phono$\operatorname{logy}{ }^{\prime}$, in N. Hornstein \& D. Lightfoot (éds.) Explanation in Linguistics. The logical problem of language acquisition. London, New York : Longman, pp. 76-115.

Dressler W. (1974). «Diachronic Puzzles for Natural Phonology », in A. Bruck, R. Fox \& M. La Galy (éds.) Papers from the parasession on Natural Phonology. Chicago : Chicago Linguistic Society, pp. 95-102.

Dressler W. (1984). «Explaining Natural Phonology», Phonology Yearbook 1 : 29-51. 
Durand J. \& Laks B. (1996). "Why Phonology is one », in J. Durand \& B. Laks (éds.) Current trends in Phonology: Models and Methods. Salford, Manchester : ESRI, pp. 3-13.

Durand J., Laks B. \& Lyche (2002). «La phonologie du français contemporain : usages, variétés et structure ", in C. Pusch \& W. Raible (éds.) Romance Corpus Linguistics Corpora and Spoken Language. Tübingen : Narr, pp. 93-106.

Durand J. \& Lyche C. (2003). «Le projet 'Phonologie du Français Contemporain' (PFC) et sa méthodologie », in E. Delais-Roussarie \& J. Durand (éds.) Corpus et variation en phonologie du français. Toulouse : Presses Universitaires du Mirail, pp. 213-276.

Dziubalska-Kołaczyk K. (2001). «Phonotactic constraints are preferences », in K. Dziubalska-Kołaczyk (éd.) Constraints and Preferences. Berlin : Mouton de Gruyter, pp. 69-100.

Encrevé P. (1997). "L'ancien et le nouveau. Quelques remarques sur la phonologie et son histoire », Langages $125: 100-123$. Version anglaise (2000) : « The Old and the New : Some Remarks on Phonology and its History ». Folia Linguistica 34 : 57-85.

Goldsmith J. (1976). Autosegmental Phonology. Thèse de doctorat, MIT.

Gordon M. (1999). Syllable Weight: Phonetics, Phonology and Typology. Thèse de doctorat, UCLA.

Goyvaerts D. (1981). "Introduction », in D. Goyvaerts (éd.) Phonology in the 1980's. Ghent : Story-Scientia, pp. 1-26.

Greenberg J. (1966). "Synchronic and Diachronic Universals in Phonology », Language 42 : 508-517.

Greenberg J. (1978). Universals of Human Language, 3 vols. Stanford : Stanford University Press.

Gurevich N. (2004). Lenition and Contrast. New York \& London : Routledge.

Gussmann E. (1980). Studies in Abstract Phonology. Cambridge Mass. : MIT Press.

Gussmann E. (2003). "Introduction to the government phonology-natural phonology workshop ». Communi- 
Présentation. En quoi la phonologie est vraiment différente

cation présentée au 34th Poznań Linguistic Meeting, Poznań 1-3 May 2003.

Hagège C. (1976). La grammaire générative: réflexions critiques. Paris : PUF.

Hale M. \& Reiss C. (2000). "Phonology as Cognition », in N. Burton-Roberts, P. Carr \& G. Docherty (éds.) Phonological Knowledge. Conceptual and Empirical Issues. Oxford : Oxford University Press, pp. 161-184.

Halle M. (1959). The Sound Pattern of Russian. The Hague: Mouton.

Hargus S. \& Kaisse E. (éds.) (1993). Studies in Lexical Phonology. New York : Academic Press.

Harris J. \& Lindsey G. (2000). " Vowel Patterns in Mind and Sound », in N. Burton-Roberts, P. Carr \& G. Docherty (éds.) Phono-logical Knowledge. Conceptual and Empirical Issues. Oxford: Oxford University Press, pp. 185-205.

Hayes B. (1981). A Metrical Theory of Stress Rules. Thèse de doctorat, MIT. Publiée par Garland Press, New York 1985.

Hayes B. (1995). Metrical Stress Theory. Principles and Case Studies. Chicago, London : University of Chicago Press.

Hayes B. (1999). «Phonetically driven phonology : the role of Optimality Theory and Inductive Grounding », in $\mathrm{M}$. Darnell, E. Moravcsik, F. Newmeyer, M. Noonan \& K. Wheatley (éds.) Functionalism and formalism in linguistics Vol.1: General papers. Amsterdam: Benjamins. ROA, pp. 243-285.

Hayes B., Kirchner R. \& Steriade D. (2004). Phonetically-Based Phonology. Cambridge : Cambridge University Press.

Hellberg S. (1978). «Unnatural phonology», Journal of Linguistics 14 : 157-177.

Hjelmslev, L. (1948). "Structural analysis of language », Studia Linguistica $1: 69-78$.

Hockett, C. (1948). " A note on structure », International Journal of American Linguistics 14 : 269-271. 
Hooper J. (1973). Aspects of Natural Generative Phonology. Thèse de doctorat, UCLA.

Hooper J. (1974). «Rule morphologization in natural generative phonology », in A. Bruck, R. Fox \& M. La Galy (éds.) Papers from the parasession on Natural Phonology. Chicago : Chicago Linguistic Society, pp. 160-170.

Hooper J. (1975). « The archi-segment in Natural Generative Phonology », Language 51 : 536-560.

Hooper J. (1976). An Introduction to Natural Generative Phonology. New York : Academic Press.

Hulst H. v. d. \& Ritter N. (2000). "The SPE-heritage of Optimality Theory », The Linguistic Review 17 : 259-289.

Hurch B. \& Rhodes R. (éds.) (1996). Natural Phonology: The State of the Art. Berlin : Mouton de Gruyter.

Hyman L. (1970). « How Concrete is Phonology? », Language $46: 58-76$.

Jackendoff R. (1997). The Architecture of the Language Faculty. Cambridge, Mass. : MIT Press.

Jackendoff R. (2002). Foundations of Language. Brain, Meaning, Grammar, Evolution. Oxford: Oxford University Press.

Joos M. (1942). «A phonological dilemma in Canadian English », Language $18: 141-144$.

Kahn D. (1976). Syllable-based generalizations in English phonology. Thèse de doctorat, MIT. Publiée par Garland Press, New York 1980.

Katz J. \& Bever T. (1974). The fall and rise of empiricism. Bloomington : Indiana University Linguistics Club. WEB.

Kaun A. (1995). The typology of rounding harmony: an Optimality Theoretic approach. Thèse de doctorat, UCLA.

Kaye J. (1989). Phonology. A cognitive view. Hillsdale : Erlbaum.

Kaye J. (1990). « What ever happened to dialect B ? », in J. Mascaró \& M. Nespor (éds.) Grammar in Progress: GLOW Essays for Henk van Riemsdijk. Dordrecht : Foris, pp. 259-263. 
Présentation. En quoi la phonologie est vraiment différente

Kaye J. (1995). «Derivations and Interfaces », in J. Durand \& F. Katamba (éds.) Frontiers of Phonology. London \& New York: Longman, pp. 289-332. Egalement in SOAS Working Papers in Linguistics and Phonetics (1993) 3 : 90-126. WEB.

Kaye J. (2000). A Users' Guide to Government Phonology. Ms. WEB.

Kaye J. (2001). "Working with licensing constraints ", in K. Dziubalska-Kołaczyk (éd.) Constraints and Preferences. Berlin \& New York: Mouton de Gruyter, pp. 251-268. WEB.

Kean M.-L. (1974). "The Strict Cycle in Phonology», Linguistic Inquiry 5 : 179-203.

Kegl, J., A. Senghas \& M. Coppola (1999). « Creation through contact: Sign language emergence and sign language change in Nicaragua ", in M. DeGraff (éd) Language creation and language change, 179-238. Cambridge, Mass. : MIT Press.

Kenstowicz M. \& Kisseberth C. (1977). Topics in Phonological Theory. New York : Academic Press.

Kenstowicz M. \& Kisseberth C. (1979). Generative Phonology. Description and Theory. San Diego : Academic Press.

Kiparsky P. (1968a/1973). "How abstract is phonology? », Publié en 1973 in O. Fujimura (éd.) Three Dimensions of Linguistic Theory. Tokyo : TEC, pp. 5-56.

Kiparsky P. (1968b). «Linguistic universals and linguistic change », in E. Bach \& R. Harms (éds.) Universals in linguistic theory. New York: Holt, Rinehart \& Winston, pp. 170-202.

Kiparsky P. (1974). "On the Evaluation Measure », in A.Bruck, R. Fox \& M. La Galy (éds.) Papers from the parasession on Natural Phonology. Chicago: Chicago Linguistic Society, pp. 328-337.

Kiparsky P. (1982a). «From Cyclic Phonology to Lexical Phonology ", in H. van der Hulst \& N. Smith (éds.) The 
structure of phonological representations. Dordrecht: Foris, vol. I, pp. 131-175.

Kiparsky P. (1982b). "Lexical phonology and morphology », in In-Seok Yang (éd.) Linguistics in the morning calm. Seoul : Hanshin, pp. 3-91.

Kiparsky P. (1982c). Explanation in Phonology. Dordrecht: Foris.

Kiparsky P. (1985). «Some Consequences of Lexical Phonology », Phonology Yearbook 2 : 85-138.

Kirchner R. (1998). An effort-based approach to consonant lenition. Thèse de doctorat, UCLA. ROA.

Koerner E. F. K. (2003). « Remarks on the origins of morphophonemics in American structuralist linguistics », Language and Communication $23: 1-43$.

Koutsoudas A. (1980). « The question of rule ordering: some common fallacies », Journal of Linguistics 16 : 19-35.

Kräuter J. F. (1877). Zur Lautverschiebung. Strassburg : Trübner.

Kučera H. (1961). The phonology of Czech. The Hague : Mouton.

Lacan J. (1966). Ecrits. Paris : Seuil.

Laks B. (1996). Langage et cognition, l'approche connexionniste. Paris : Hermès.

Laks B. (1998). « Le connexionnisme, les représentations et la question des niveaux », Praxiling $31:$ 149-176.

Laks B. (2005). "Approches cognitives de la phonologie », in J. Durand, N. Nguyen, V. Rey \& S. Wauquier-Gravelines (éds.) Phonologie et phonétique: approches contemporaines. Paris : Hermes.

Laks B. (à paraître). «La phonologie générative naturelle et la phonologie naturelle », in S. Auroux, E.F.K. Koerner, H.-J. Niederehe \& K. Versteegh (éds.) History of the Language Sciences, vol.3. Berlin : Mouton de Gruyter.

Lass R. (1984). Phonology. An introduction to basic concepts. Cambridge : Cambridge University Press.

Leben W. \& Robinson O. (1977). «Upside-down Phonology », Language $53: 1-20$. 
Présentation. En quoi la phonologie est vraiment différente

Lehiste I. (1960). An Acoustic-Phonetic Study of Internal Open Juncture. Basel, New York: Karger (supplément à Phonetica 5).

Lévi-Strauss C. (1958). Anthropologie structurale. Paris : Plon.

Lightner T. $\left(1978^{2}\right)$. «Generative Phonology», in W. Orr Dingwall (éd.) A Survey of Linguistic Science. $2^{\text {ème }}$ édition Stamford, Connecticut : Greylock, pp. 1-32.

Lightner T. (1981). «New explorations into Derivational Morphology », in D. Goyvaerts (éd.) Phonology in the 80's. Ghent : Story-Scientia, pp. 93-99.

Lowenstamm J. (1996). " CV as the only syllable type », in J. Durand \& B. Laks (éds.) Current trends in Phonology. Models and Methods. Salford, Manchester : ESRI, pp. 419441.

Mascaró J. (1976). Catalan Phonology and the Phonological Cycle. Thèse de doctorat,. MIT.

McCawley J. (1968). The Phonological Component of a Grammar of Japanese. The Hague : Mouton.

McGarrity L. (2003). Constraints on patterns of primary and secondary stress. Thèse de doctorat, Université d'Indiana.

McMahon A. (2000). Lexical Phonology and the history of English. Cambridge : Cambridge University Press.

Meyer-Benfey H. (1901). « Über den Ursprung der germanischen Lautverschiebung », Zeitschrift für deutsches Altertum und deutsche Litteratur 45 : 101-128.

Milner J.-C. (1989). Introduction à une science du langage. Paris : Seuil.

Mohanan K. (1982). Lexical Phonology. Thèse de doctorat, MIT.

Mohanan K. (1986). The Theory of Lexical Phonology. Dordrecht : Reidel.

Morelli F. (1999). The phonotactics and phonology of obstruent clusters in Optimality Theory. Thèse de doctorat, Université du Maryland.

Newmeyer F. (1998). "The irrelevance of typology for linguistic theory », Syntaxis $1: 161-197$. 
Paul H. (1880). Pinzipien der Sprachgeschichte. $9^{\mathrm{e}}$ édition 1975 Tübingen : Niemeyer.

Plénat M., Lignon S., Serna N. \& Tanguy L. (2002). «La conjecture de Pichon », Corpus 1 : 105-150.

Rubach J. (1984). Cyclic and Lexical Phonology: The Structure of Polish. Dordrecht : Foris.

Rubach J. (1993). The Lexical Phonology of Slovak. Oxford: Clarendon Press.

Rubach J. \& Booij G. (1984). "Morphological and prosodic domains in Lexical Phonology », Phonology 1 : 1-27.

de Saussure F. (1915). Cours de linguistique générale. Paris 1972 : Payot.

Schane S. (1968). French Phonology and Morphology. Cambridge, Mass. : MIT Press.

Schane S. (1974). «How abstract is abstract? », in A. Bruck, R. Fox \& M. La Galy (éds.) Papers from the parasession on Natural Phonology. Chicago : Chicago Linguistic Society, pp. 297-317.

Scheer T. (1999). «A theory of consonantal interaction », Folia Linguistica 32 : 201-237. WEB.

Scheer T. (2003). "Privative melodic representations and structuralist thinking: Licensing Constraints $»$. Communication présentée au colloque du PFC, Paris 11-13 décembre 2003. WEB.

Scheer T. (2004). A Lateral Theory of Phonology. Vol.1: What is $C V C V$, and why should it be? Berlin : Mouton de Gruyter.

Scheer T. (ms). A Lateral Theory of Phonology. Vol.2: On Locality, Morphology and Phonology in Phonology. Berlin : Mouton de Gruyter.

Scobbie J., Coleman J. \& Bird S. (1996). «Key aspects of declarative phonology », in J. Durand \& B. Laks (éds.) Current Trends in Phonology: Models and Methods. Salford, Manchester : ESRI, Vol.2, pp. 685-709.

Skinner F. (1957). Verbal Behaviour. Acton, MA : Copley.

Stampe D. (1972). How I Spent my Summer Vacation. Thèse de doctorat, Université de Chicago. 
Présentation. En quoi la phonologie est vraiment différente

Steriade D. (1987). "Locality Conditions and Feature Geometry », in J. McDonough \& B. Plunkett (éds.) Proceedings of NELS 17. Amherst: GLSA, pp. 595-617.

Steriade D. (1995). «Underspecification and Markedness », in J. Goldsmith (éd.) The Handbook of Phonological Theory. Oxford : Blackwell, pp. 114-174.

Steriade D. (1997). Phonetics in Phonology: The case of Laryngeal Neutralization. Ms, UCLA.

Steriade D. (2003). "Choosing a base of affixation". Communication présentée à la CASTL kick-off conference, Tromsø 30 September 2003.

Szigetvári P. (1999). VC Phonology : a theory of consonant lenition and phonotactics. Thèse de doctorat. Université Eötvös Loránd, Budapest. Disponible à http://seas3.elte.hu/ szigetva/papers.html.

Tranel B. (1981). Concreteness in Generative Phonology. Evidence from French. Berkeley : Univ. of California Press.

Vennemann T. (1971). "The phonology of Gothic vowels ", Language 47 : 90-132.

Vennemann T. (1972a). «On the theory of syllabic phonology », Linguistische Berichte 18 : 1-18.

Vennemann T. (1972b). "Sound change and markedness theory: On the history of the German consonant system », in R.P. Stockwell \& R.K.S. Macaulay (éds.) Linguistic change and generative theory. Essays from the UCLA Conference on historical linguistics in the perspective of transformational theory (1969). Bloomington: Indiana Univ. Press, pp. 230-274.

Vennemann T. (1974a). «Phonological concreteness in natural generative grammar», in R. Shuy \& C. Bailey (éds.) Toward tomorrow's linguistics. Washington, DC: Georgetown University Press, pp. 202-219.

Vennemann T. (1974b). "Words and syllables in natural generative grammar », in A. Bruck, R. Fox \& M. La Galy (éds.) Papers from the parasession on Natural Phonology. Chicago : Chicago Linguistic Society, pp. 346-374. 
Vennemann T. (1976). «Vowel alternations in English, German and Gothic: Remarks on realism in phonology », in $\mathrm{M}$. Jazayery, E. Polome \& W. Winter (éds.) Linguistic and Literary Studies in Honor of Archibald A. Hill, vol. I : General and Theoretical Linguistics. Lisse : Ridder, pp. 337-359.

Vennemann T. (1978). "Universal Syllabic Phonology», Theoretical Linguistics 5 : 175-215.

Vennemann T. (1984). "Hochgermanisch und Niedergermanisch. Die Verzweigungstheorie der germanischdeutschen Lautverschiebungen », Beiträge zur Geschichte der deutschen Sprache und Literatur 106 : 1-45.

Vennemann T. (1988). Preference laws for syllable structure and the explanation of sound change. Berlin : Mouton de Gruyter.

Vennemann T. (1991). "Syllable structure and syllable cut prosodies in Modern Stadard German », in P. M. Bertinetto, M. Kenstowicz \& M. Loporcaro (éds.) Certamen Phonologicum II. Torino : Rosenberg \& Sellier, pp. 211-243.

Vennemann T. (1994). "Linguistic reconstruction in the context of European prehistory », Transactions of the Philological Society $92: 215-284$.

Vennemann T. (1997). «The development of reduplicating verbs in Germanic », in I. Rauch \& G. Carr (éds.) Insights in Germanic Linguistics II. Berlin, New York : de Gruyter, pp. 297-336.

Walker R. (1998). Nasalization, neutral segments and opacity effects. Thèse de doctorat, Univ. de Californie à Santa Cruz.

Wauquier, S. (en préparation). Des représentations et procédures en phonologie : acquisition, traitement de la parole continue et dysphasie développementale. Habilitation à diriger des recherches, EHESS.

Wells R. (1947). «Immediate Constituents », Language 23 : 81-117.

Zhang J. (2001). The Effects of Duration and Sonority on Contour Tone Distribution - Typological Survey and Formal Analysis. Thèse de doctorat, UCLA. 